\title{
Electromagnetic response to high-frequency gravitational waves having additional polarization states: distinguishing and probing tensor-mode, vector-mode and scalar-mode gravitons
}

\author{
Fang-Yu Li ${ }^{1, a}$, Hao Wen ${ }^{1, b}$, Zhen-Yun Fang ${ }^{1}$, Di Li ${ }^{2}$, Tong-Jie Zhang ${ }^{3}$ \\ ${ }^{1}$ Physics Department, Chongqing University, Chongqing 401331, China \\ ${ }^{2}$ National Astronomical Observatories, Chinese Academy of Science, A20 Datun Road, Chaoyang District, Beijing 100012, China \\ ${ }^{3}$ Department of Astronomy, Beijing Normal University, Beijing 100875, China
}

Received: 6 November 2018 / Accepted: 3 September 2020 / Published online: 22 September 2020

(C) The Author(s) 2020

\begin{abstract}
Gravitational waves (GWs) from extra dimensions, very early universe, and some high-energy astrophysical processes might have at most six polarization states: tensor- and nontensor-mode gravitons. The peak regions or partial peak regions (of the amplitudes or energy densities) of some of such GWs are just distributed in the GHz or higher frequency band, which would be an optimal frequency band for the electromagnetic (EM) response to such highfrequency GWs (HFGWs). In this paper we investigate the EM response to the HFGWs, and for the first time we obtain the concrete form of analytic solutions of the perturbative EM fields caused by all six possible polarizations of the HFGWs in the background stable EM fields and in the proposed three dimensional synchro-resonance system (3DSR system), respectively. It is found that all such six polarizations may in principle show separability and detectability. Moreover, the detection frequency band $\left(\sim 10^{8}\right.$ to $10^{12} \mathrm{~Hz}$ or higher) of the signal photon fluxes by the 3DSR system and the observation frequency range $\left(\sim 7 \times 10^{7}\right.$ to $3 \times 10^{9}$ $\mathrm{Hz}$ ) of the signals by the FAST (Five-hundred-meter Aperture Spherical Telescope, China) have a certain overlapping property, and thus their coincidence experiments in the future for observations will have high complementarity.
\end{abstract}

\section{Introduction}

Recently, the LIGO Scientific Collaboration and the Virgo Collaboration reported multiple gravitational wave (GW) evidence (GW150914, GW151226, GW170104, GW170608, GW170814, GW170817) [1-8] and a candidate (LVT151012) [8]. These GW events and candidate are mainly produced by

\footnotetext{
a e-mail: fangyuli@cqu.edu.cn (corresponding author)

be-mail: wenhao@cqu.edu.cn (corresponding author)
}

binary black hole mergers (frequencies around $30-450 \mathrm{~Hz}$, dimensionless amplitudes in the region of the Earth around $h \sim 10^{-21}$ to $h \sim 10^{-22}$ ), and one event of them is the detection evidence of GWs produced by a binary neutron star merger [6]. Obviously, these are the most important achievements since the observation of an indirect effect of gravitational radiation from PSR1913+16 for GW projects. The binary neutron star merger was accompanied by gamma-ray radiation almost at the same time, which is an electromagnetic (EM) counterpart of the GWs. In fact, the EM counterparts and the EM signals caused by the effect of EM response to GWs, may exist widely in the universe, and thus they will provide a new and effective tool for observation and detection of the GWs. Obviously, the above-mentioned results provide direct evidence for the detection of the GWs expected by the GR. Moreover, these achievements have also following high scientific significance:

1. The amplitude magnitude of $h \sim 10^{-21}$ to $h \sim 10^{-22}$ for such GWs shows the rationality of the weak field linear approximation and the perturbation theory of gravity. Thus it further increases the possibility and hope of searching gravitons of spin- 2 in the quantization process of gravity.

2. The information (including the related energymomentum) carried by the GWs from the wave sources of the binary compact objects also provides strong evidence of the positive definite property of the energy-momentum tensor for the GW fields themselves.

3. Observation of the GWs emitted by binary neutron star merger and the related EM counterpart (gamma-ray radiation) gives a strict limit to the propagating velocity of the GWs in the intermediate frequency band $(\sim 1-1000 \mathrm{~Hz})$. Also, it provides an effective constraint for the geometry of extra dimensions $[9,10]$. 
On the other hand, the results obtained by LIGO and Virgo should not mean the end of seeking the GWs. On the contrary, they are just the beginning of the GW astronomy. This is because of the following reasons:

1. With improvement of the sensitivities of LIGO, Virgo, GEO, KAGRA, AIGO etc., the searchable sky area and detectable space scale will be further expanded. Therefore, it is very well possible that more $\mathrm{GW}$ evidence will be detected and found during the next decade.

2. The GWs recently detected by the LIGO and Virgo Collaborations are located in an interesting but special intermediate frequency range $(v \sim 30$ to $\sim 450 \mathrm{~Hz})$, and their durations of the signals in the detectors were very short. Thus, observation and detection of the continuous GWs, other kinds of GWs and the GWs in other frequency bands will be urgent affairs.

3. Except for the GWs predicted by General Relativity (GR), series of modified gravity theories and the gravity theories beyond GR also expect the GWs [9,11-21]. An important difference from the GR is that the GWs in some of such gravity theories might have additional polarization states, which can be at most six polarization states in our $3+1$ dimensional spacetime, while the GWs in the GR have only two polarization states $(\oplus$-type and $\otimes$-type). Thus, further theoretical study and experimental observation of the GWs will provide important criterion for the polarization states, the propagating speed [3], the waveforms, and other possible novel properties of the GWs.

For the intermediate frequency GWs $\left(v \sim 1\right.$ to $v \sim 10^{3}$ $\mathrm{Hz}$ ), Nishizawa et al. investigated effective methods and schemes to detect and separate the different polarization states of such GWs. In fact, such schemes are based on the extended method of correlation analysis for the nontensorial polarizations [22,23], and they proposed the scheme to use a network of ground-based laser interferometers (with the pulsar timing array). Such a network with more than three detectors can separate the mixture of polarization modes in detector outputs, and they have almost the same sensitivity to the tensor, vector and scalar polarization mode of the stochastic GW background. This scheme is only suitable for the intermediate frequency in $1-1000 \mathrm{~Hz}$, i.e., it is not suitable for the HFGWs in the microwave frequency band. In comparison, our scheme is to distinguish and display different polarization states of the HFGWs in GHz or higher bands by the EM response. Thus, they have good mutual complementarity in terms of frequency band, method and wave source. For the detection of low-frequency GWs ( $v \sim 1$ to $\sim 10^{-7} \mathrm{~Hz}$ ), a related scheme is based on the configuration of space-based GW detectors [24]. Obviously, the above schemes are based on the tidal action caused by these polarization states of the GWs, and they would be promising for displaying and separating all polarizations. Moreover, Visinalli et al. studied the scheme probing extra dimensions by intermediate frequency
GW signals and related EM counterparts from the mergers of binary neutron stars [10]. Such a scheme and direct detection of the additional polarizations of the HFGWs from the extra dimensions, will be complementary in terms of frequency band and principle.

On the other hand, almost all mainstream inflationary theories and universe models predicted the primordial (relic) GWs, with spectrum distributed in a very wide frequency region, which may be from extreme-low-frequency range ( $\sim 10^{-16}$ to $\left.\sim 10^{-17} \mathrm{~Hz}\right)$ to high-frequency band $\left(\sim 10^{8}\right.$ to $\sim 10^{10} \mathrm{~Hz}$ or higher) [25-32]. Especially, the peak region or partial peak region of the energy densities of the relic HFGWs predicted by the pre-big-bang models [27,28], the quintessential inflationary models [29-31], and the shortterm anisotropic inflation model [32], are just distributed in the typical microwave band $\left(v \sim 10^{8}\right.$ to $\sim 10^{10}$ $\mathrm{Hz}$ ), in which corresponding amplitudes might reach up to $h \sim 10^{-26}$ to $h \sim 10^{-30}$. Moreover, the frequency of the HFGWs (KK-gravitons) expected by the braneworld scenarios $[9,21]$ from extra dimensions and the HFGWs predicated by the interaction between astrophysical plasma and intense electromagnetic waves (EMWs) [33] has been extended to $\sim 10^{9}$ to $\sim 10^{12} \mathrm{~Hz}$ or higher (related amplitudes of these HFGWs would be expected to be $h \sim 10^{-21}$ to $\sim 10^{-27}$ $[9,21,33])$, and some work predicted HFGWs in the very high-frequency band even over $10^{19} \mathrm{~Hz}$ from coherent oscillation of electron-positron pairs and fields [34] or from magnetars [35]. Another important possible source of HFGWs is produced during the preheating in the early universe and it would have a frequency over GHz [36,37]. Mergers [38] and evaporation [39] of primordial black holes are also possible HFGW sources, and their frequency band can be $\sim 10^{9}$ to $10^{13} \mathrm{~Hz}$, in which the related amplitudes might be $h \sim 10^{-31}$ to $h \sim 10^{-36}$, respectively. In fact, according to the GR, and even modified gravity theories and the gravity theories beyond the GR, any energy-momentum tensor in the highfrequency oscillating states with deviation from the spherical symmetry or cylindrical symmetry would be possible and potential HFGW sources. Thus such mechanisms and process would be very common in high-energy astrophysics and cosmology, and these HFGWs would contain abundant astrophysical and cosmological information.

Among the above possible HFGW sources, in this article we will particularly put attention on those sources (e.g. see Refs. $[9,21])$ which would contribute additional polarizations (vector-mode, scalar-mode) of the HFGWs.

However, the frequencies of these HFGWs are far beyond the detection or observation range of intermediate-frequency GWs ( $\sim 1$ to $\sim 10^{3} \mathrm{~Hz}$, by ground-based GW detectors), the low-frequency GWs $\left(\sim 1\right.$ to $\sim 10^{-7} \mathrm{~Hz}$, by space GW detectors) and the extreme-low-frequency GWs $\left(\sim 10^{-16}\right.$ to $\sim 10^{-17} \mathrm{~Hz}$, by B-mode polarization in the CMB). Therefore, detection and observation of the HFGWs (including 
separation of the additional polarization states) need a new principle and scheme. So far, the separation and distinguishing of the additional polarization states of the HFGWs in the microwave frequency band by the EM response almost have not been reported in the past.

In this paper, based on the electrodynamics and the quantum electronics in curved spacetime, we investigate a novel way to distinguish and display all possible six polarization states of the HFGWs. We will address the concrete forms of analytic solutions for the perturbative EM fields caused by all six possible polarizations of the HFGWs in the background stable EM fields, and study the perturbative EM signals of the HFGWs with additional polarizations in the proposed 3DSR system [40-44] (in laboratory scale) and in galacticextragalactic background EM fields. The 3DSR plan is performed in China by cooperations of universities and institutes from several countries. The 3DSR detector would be able to observe and distinguish the EM signals (presented in this work) caused by all possible six polarizations of the HFGWs, based on very different physical behaviors between the signal photons and the noise background, especially by their very different strength distributions, propagating directions, spectrum, decaying rates and wave impedances (see Sect. 5).

Our attention will focus mainly on the EM response to the HFGWs from extra dimensions, from the very early stage of the universe and from some high-energy astrophysical process, especially the HFGWs (KK-gravitons) from the braneworld $[9,21]$, the relic HFGWs from the short-term anisotropic inflation [32], from the pre-big-bang $[27,28]$, from the quintessential inflation [29-31], and the HFGWs from the astrophysical plasma oscillation [33]. This is because they involve the following important scientific issues and problems: the extra dimensions of space and the brane universes, the very early universe and inflationary epoch, the start point of time or the information from the pre-big-bang, the essence and candidates of dark energy, and the interaction mechanism of the astrophysical plasma with intense EM radiations. So far, the high frequency band GWs have not yet been detected by such EM responses (or by any other method). However, we think it is interesting to carry out the theoretical research on relevant effects before the experiments, and it would also provide some theoretical predictions and reference for the construction of detectors and potential observations in the future.

The plan of this paper is as follows. In Sect. 2, we shall show the general form of the HFGWs having six polarization states. In Sect. 3, we address the analytic solutions for the perturbative EM fields caused by six polarization states of HFGWs in the background stable EM fields. In Sect. 4, we study the EM signals caused by the six polarizations of HFGWs in the 3DSR system (in laboratory scale), and their separation and displaying effects. In Sect. 5, numerical esti- mations of the perturbative photon fluxes in the 3DSR system and in the galactic-extragalactic magnetic fields are given. Our conclusion is summarized in Sect. 6.

\section{High-frequency gravitational wave (HFGWs) having additional polarization states}

1. In general, the "monochromatic components" of the GWs having six polarization states and propagating along the $z$ direction can be written as

$$
h_{\mu \nu}=\left(\begin{array}{cccc}
0 & 0 & 0 & 0 \\
0 & A_{\oplus}+A_{b} & A_{\otimes} & A_{x} \\
0 & A_{\otimes} & -A_{\oplus}+A_{b} & A_{y} \\
0 & A_{x} & A_{y} & \sqrt{2} A_{l}
\end{array}\right) e^{i\left(k_{g} z-\omega_{g} t\right)},
$$

where $\oplus, \otimes, \mathrm{x}, \mathrm{y}, \mathrm{b}$ and $l$ represent $\oplus$-type, $\otimes$-type polarizations (tensor-mode gravitons), $\mathrm{x}$-type, y-type polarizations (vector-mode gravitons), b-type and $l$-type polarizations (scalar-mode gravitons), respectively. For the coherent and non-stochastic GWs, such as the GWs from extra dimensions (e.g. K-K GWs from braneworld [9,21]), the $A_{\oplus}, A_{\otimes}$, $A_{x}, A_{y}, A_{b}$ and $A_{l}$ are constant values of amplitudes of the GWs in the laboratory frame of reference. For the relic GWs, $A_{\oplus}, A_{\otimes}, A_{x}, A_{y}, A_{b}$ and $A_{l}$ are stochastic values of the amplitudes of the relic GWs in the laboratory frame of reference, which contain the cosmology scale factor; $k_{g}$ and $\omega_{g}$ are wave number and angular frequency of the GWs, respectively. Equation (1) can be conveniently represented in the following matrix form [22]:

$$
\begin{aligned}
e_{i j}^{\oplus}=\left(\begin{array}{ccc}
1 & 0 & 0 \\
0 & -1 & 0 \\
0 & 0 & 0
\end{array}\right), & e_{i j}^{\otimes}=\left(\begin{array}{lll}
0 & 1 & 0 \\
1 & 0 & 0 \\
0 & 0 & 0
\end{array}\right) \\
e_{i j}^{x}=\left(\begin{array}{lll}
0 & 0 & 1 \\
0 & 0 & 0 \\
1 & 0 & 0
\end{array}\right), & e_{i j}^{y}=\left(\begin{array}{lll}
0 & 0 & 0 \\
0 & 0 & 1 \\
0 & 1 & 0
\end{array}\right) \\
e_{i j}^{b}=\left(\begin{array}{lll}
1 & 0 & 0 \\
0 & 1 & 0 \\
0 & 0 & 0
\end{array}\right), & e_{i j}^{l}=\sqrt{2}\left(\begin{array}{lll}
0 & 0 & 0 \\
0 & 0 & 0 \\
0 & 0 & 1
\end{array}\right) .
\end{aligned}
$$

2. Phase modification issues in the EM response to the HFGWs.

We have previously showed that (Sect. III of Ref. [45]), although the gravitons may have extremely small but nonvanishing masses, the phase modification caused by the difference between the phase velocity $v_{P}$ of the HFGWs (gravitons) and the phase velocity $c$ of the EMW (photon fluxes) can often be neglected even for a large propagation range. This means that even if the propagating distance of the HFGWs into the background stable EM fields reaches up to typical astrophysical size (e.g. $\Delta z \sim 10^{21} \mathrm{~m}$ ), such a modification 
will not impact the generation and accumulation effect of the perturbative EM waves.

3. The metric components of the HFGWs having six polarization states.

It is well known that, for weak GWs, the metric can often be expressed as a small perturbation to the background spacetime $\eta_{\mu \nu}$, i.e.,

$g_{\mu \nu}=\eta_{\mu \nu}+h_{\mu \nu}$

From Eqs. (1) and (2), it is easy to give the covariant components of the metric tensor for the HFGWs, and we have

$$
\begin{aligned}
\operatorname{det}\left|g_{\mu \nu}\right|= & 1+h_{11}+h_{22}+h_{33} \\
& +O\left(h^{2}\right) \approx 1+2 h_{b}+h_{l} .
\end{aligned}
$$

In the above expressions, we neglected the second-order infinite small quantity $h^{2}$ of the perturbation $h_{i j}$.

Obviously, the HFGWs expressed by Eqs. (1) and (2) do not satisfy the transverse and traceless gauge condition (TTgauge condition). Different polarization states in Eq. (1) correspond to different kinds of gravitons, where the $\oplus$-type and $\otimes$-type polarization states represent gravitons of spin-2; the $\mathrm{x}$-type and y-type polarization states indicate gravitons of spin- 1 , and the b-type and $l$-type polarization states denote gravitons of spin- 0 , respectively.

In fact, the EM perturbation effects produced by the HFGWs and their detections had been discussed. These EM detection systems include constructed and proposed ones like the two coupled spherical cavities [46], high-frequency phonon trapping acoustic cavities [47], closed cylindrical superconducting cavity [48] and coupling system between the planar superconducting open cavity and static magnetic field [49]. However, in this previous research, the HFGWs interacting with such EM systems are almost restricted to GWs in the GR framework, i.e., they satisfy the TT-gauge condition. So far, it is not clear what a concrete form (of the EM response to the HFGWs having additional polarization states) should be; it is also not clear whether such different polarization states of the HFGWs can be separated and displayed, and how to separate and display them. We shall show these issues in the expanded EM systems (see Sect. 4), which consist not only of static magnetic fields, but also of static electric fields. Moreover, they also include the coupling system between the Gaussian-type microwave photon flux (Gaussian beam) and the static high EM fields. Then the EM perturbative effects produced by the different polarization states of the HFGWs will have different physical behaviors. Therefore, these different polarization states, in principle, can be separated and displayed.

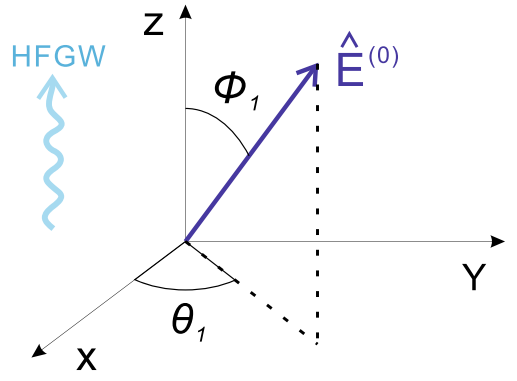

Fig. 1 The background stable electric field $\hat{E}^{(0)}$ has an arbitrary direction. Here the HFGW propagates along the $z$-direction

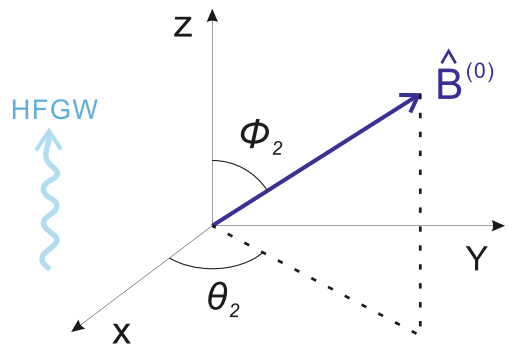

Fig. 2 The background stable magnetic field $\hat{B}^{(0)}$ has also an arbitrary direction. This magnetic field and the background electric field in Fig. 1 are in the same region as the region that the HFGW passes through, and the HFGW propagates along the $z$-direction

\section{The perturbative effects of the HFGWs having six polarization states to the background stable EM fields}

1. The perturbative solutions in the stable EM fields.

In the EM response, the electric and magnetic fields may be present at the same time, and they can point in arbitrary directions (see Figs. 1 and 2).

In Eqs. (5), (10) and Figs. 1, 2, the " $\wedge$ " stands the stable EM fields, and superscript "0" denotes the background EM fields. From Figs. 1 and 2, the background stable EM fields can be written in the following component forms:

$$
\begin{aligned}
& \hat{E}_{x}^{(0)}=\hat{E}^{(0)} \sin \theta_{1} \cos \phi_{1}, \hat{E}_{y}^{(0)}=\hat{E}^{(0)} \sin \theta_{1} \sin \phi_{1}, \\
& \hat{E}_{z}^{(0)}=\hat{E}^{(0)} \cos \theta_{1}, \hat{B}_{x}^{(0)}=\hat{B}^{(0)} \sin \theta_{2} \cos \phi_{2}, \\
& \hat{B}_{y}^{(0)}=\hat{B}^{(0)} \sin \theta_{2} \sin \phi_{2}, \hat{B}_{z}^{(0)}=\hat{B}^{(0)} \cos \theta_{2},
\end{aligned}
$$

According to Eq. (5), the covariant and the contra-variant components of the background EM field tensor are given (we use MKS units) by $F_{01}^{(0)}=-F_{10}^{(0)}=-\frac{1}{c} \hat{E}_{x}^{(0)}, F_{02}^{(0)}=$ $-F_{20}^{(0)}=-\frac{1}{c} \hat{E}_{y}^{(0)}, F_{03}^{(0)}=-F_{30}^{(0)}=-\frac{1}{c} \hat{E}_{z}^{(0)}, F_{12}^{(0)}=$ $-F_{21}^{(0)}=\hat{B}_{z}^{(0)}, F_{13}^{(0)}=-F_{31}^{(0)}=-\hat{B}_{y}^{(0)}, F_{23}^{(0)}=-F_{32}^{(0)}=$ $\hat{B}_{x}^{(0)}$, and

$F^{\mu \nu(0)}=\eta^{\mu \alpha} \eta^{\nu \beta} F_{\alpha \beta}^{(0)}$.

Interaction of the HFGWs, Eq. (1), with such background EM fields, Eqs. (5) and (6), will generate the EM perturbation, and the perturbative effects can be calculated by the 
electrodynamics equations in curved spacetime:

$$
\begin{aligned}
& \frac{1}{\sqrt{-g}} \frac{\partial}{\partial x^{\nu}}\left[\sqrt{-g} g^{\mu \alpha} g^{\nu \beta}\left(F_{\alpha \beta}^{(0)}+\tilde{F}_{\alpha \beta}^{(1)}\right)\right]=\mu_{0} J^{\mu}, \\
& \nabla_{\mu} F_{\nu \alpha}+\nabla_{\nu} F_{\alpha \mu}+\nabla_{\alpha} F_{\mu \nu}=0, \\
& \text { where, } \nabla_{\alpha} F_{\mu \nu}=F_{\mu \nu, \alpha}-\Gamma_{\mu \alpha}^{\sigma} F_{\sigma \nu}-\Gamma_{\nu \alpha}^{\sigma} F_{\mu \sigma} ;
\end{aligned}
$$

$\Gamma_{\mu \nu}^{\alpha}$ is the Christoffel connection of the second kind, and $J^{\mu}$ indicates the four-dimensional electric current density.

For the EM perturbation in the free space (vacuum), it has neither a real four-dimensional electric current nor other equivalent electric current, so $J^{\mu}=0$ in Eq. (7). Moreover, for the EM perturbation generated by the weak HFGWs, we have $F_{\mu \nu}=\hat{F}_{\mu \nu}^{(0)}+\tilde{F}_{\mu \nu}^{(1)}$, where $\hat{F}_{\mu \nu}^{(0)}$ is the background EM field tensor, Eqs. (5) and (6), and $\tilde{F}_{\mu \nu}^{(1)}$ is the first-order perturbation to $F_{\mu \nu}^{(0)}$; the " $\sim$ " represents time-dependent perturbative EM fields. Here we neglected the second-order and higher-order infinite small perturbations. In this case, we have

$F_{\mu \nu}=\hat{F}_{\mu \nu}^{(0)}+\tilde{F}_{\mu \nu}^{(1)}+O\left(h^{2}\right)$.

Introducing Eqs. (1), (2), (5) and (6) into Eqs. (7), (8), we obtain the following inhomogeneous hyperbolic equations:

$$
\begin{aligned}
& \square \tilde{E}_{x}^{(1)}=\frac{\partial^{2} \tilde{E}_{x}^{(1)}}{\partial z^{2}}-\frac{1}{c^{2}} \frac{\partial^{2} \tilde{E}_{x}^{(1)}}{\partial t^{2}} \\
& =k_{g}^{2}\left\{\left(h_{\oplus}-\frac{1}{2} h_{l}\right) \hat{E}_{x}^{(0)}+h_{\otimes} \hat{E}_{y}^{(0)}+h_{x} \hat{E}_{z}^{(0)}\right\} \\
& +c k_{g}^{2}\left\{h_{\otimes} \hat{B}_{x}^{(0)}-\left(h_{\oplus}+\frac{1}{2} h_{l}\right) \hat{B}_{y}^{(0)}\right. \\
& \left.+h_{y} \hat{B}_{z}^{(0)}\right\} \text {, } \\
& \square \tilde{B}_{y}^{(1)}=\frac{\partial^{2} \tilde{B}_{y}^{(1)}}{\partial z^{2}}-\frac{1}{c^{2}} \frac{\partial^{2} \tilde{B}_{y}^{(1)}}{\partial t^{2}} \\
& =k_{g}^{2} / c\left\{\left(h_{\oplus}-\frac{1}{2} h_{l}\right) \hat{E}_{x}^{(0)}+h_{\otimes} \hat{E}_{y}^{(0)}+h_{x} \hat{E}_{z}^{(0)}\right\} \\
& +k_{g}^{2}\left\{h_{\otimes} \hat{B}_{x}^{(0)}-\left(h_{\oplus}\right.\right. \\
& \left.\left.+\frac{1}{2} h_{l}\right) \hat{B}_{y}^{(0)}+h_{y} \hat{B}_{z}^{(0)}\right\} \text {, } \\
& \square \tilde{E}_{y}^{(1)}=\frac{\partial^{2} \tilde{E}_{y}^{(1)}}{\partial z^{2}}-\frac{1}{c^{2}} \frac{\partial^{2} \tilde{E}_{y}^{(1)}}{\partial t^{2}} \\
& =k_{g}^{2}\left\{h_{x} \hat{E}_{x}^{(0)}-\left(h_{\oplus}+\frac{1}{2} h_{l}\right) \hat{E}_{y}^{(0)}+h_{y} \hat{E}_{z}^{(0)}\right\} \\
& -c k_{g}^{2}\left\{\left(h_{b}-h_{\oplus}+h_{l}\right) \hat{B}_{x}^{(0)}\right. \\
& \left.+h_{\otimes} \hat{B}_{y}^{(0)}+h_{x} \hat{B}_{z}^{(0)}\right\}, \\
& \square \tilde{B}_{x}^{(1)}=\frac{\partial^{2} \tilde{B}_{x}^{(1)}}{\partial z^{2}}-\frac{1}{c^{2}} \frac{\partial^{2} \tilde{B}_{x}^{(1)}}{\partial t^{2}} \\
& =-k_{g}^{2} / c\left\{h_{x} \hat{E}_{x}^{(0)}-\left(h_{\oplus}+\frac{1}{2} h_{l}\right) \hat{E}_{y}^{(0)}+h_{y} \hat{E}_{z}^{(0)}\right\} \\
& +k_{g}^{2}\left\{\left(h_{b}-h_{\oplus}+h_{l}\right) \hat{B}_{x}^{(0)}\right. \\
& \left.+h_{\otimes} \hat{B}_{y}^{(0)}+h_{x} \hat{B}_{z}^{(0)}\right\},
\end{aligned}
$$

and, $\frac{\partial \tilde{E}_{z}^{(1)}}{\partial t}=\frac{\partial h_{x}}{\partial t} \hat{E}_{x}^{(0)}+\frac{\partial h_{y}}{\partial t} \hat{E}_{y}^{(0)}-\frac{\partial}{\partial t}\left(h_{b}-\frac{1}{2} h_{l}\right) \hat{E}_{z}^{(0)}$,

$$
\begin{aligned}
\frac{\partial \tilde{E}_{z}^{(1)}}{\partial z} & =\frac{\partial h_{x}}{\partial z} \hat{E}_{x}^{(0)}+\frac{\partial h_{y}}{\partial z} \hat{E}_{y}^{(0)}-\frac{\partial}{\partial z}\left(h_{b}-\frac{1}{2} h_{l}\right) \hat{E}_{z}^{(0)}, \\
\frac{\partial \tilde{B}_{z}^{(1)}}{\partial t} & =\frac{\partial \tilde{B}_{z}^{(1)}}{\partial z}=0,
\end{aligned}
$$

where " $\square$ " indicates the d'Alembertian.

From Eqs. (11) to (15), after lengthy calculations, we obtain the general solutions of these equations as follows:

$$
\begin{aligned}
\tilde{E}_{x}^{(1)}= & -\frac{i}{2} k_{g} z\left\{\left(h_{\oplus}-\frac{1}{2} h_{l}\right) \hat{E}_{x}^{(0)}+h_{\otimes} \hat{E}_{y}^{(0)}+h_{x} \hat{E}_{z}^{(0)}\right\} \\
& -\frac{i}{2} k_{g} c z\left\{h_{\otimes} \hat{B}_{x}^{(0)}-\left(h_{\oplus}+\frac{1}{2} h_{l}\right) \hat{B}_{y}^{(0)}+h_{y} \hat{B}_{z}^{(0)}\right\} \\
& +C_{1} \exp \left[i\left(k_{g} z+\omega_{g} t\right)\right], \\
\tilde{B}_{y}^{(1)}= & -\frac{i}{2} \frac{k_{g} z}{c}\left\{\left(h_{\oplus}-\frac{1}{2} h_{l}\right) \hat{E}_{x}^{(0)}+h_{\otimes} \hat{E}_{y}^{(0)}+h_{x} \hat{E}_{z}^{(0)}\right\} \\
& -\frac{i}{2} k_{g} z\left\{h_{\otimes} \hat{B}_{x}^{(0)}-\left(h_{\oplus}+\frac{1}{2} h_{l}\right) \hat{B}_{y}^{(0)}+h_{y} \hat{B}_{z}^{(0)}\right\} \\
& +C_{2} \exp \left[i\left(k_{g} z+\omega_{g} t\right)\right], \\
\tilde{E}_{y}^{(1)}= & -\frac{i}{2} k_{g} z\left\{h_{x} \hat{E}_{x}^{(0)}-\left(h_{\oplus}+\frac{1}{2} h_{l}\right) \hat{E}_{y}^{(0)}+h_{y} \hat{E}_{z}^{(0)}\right\} \\
& +\frac{i}{2} k_{g} c z\left\{\left(h_{b}-h_{\oplus}+h_{l}\right) \hat{B}_{x}^{(0)}+h_{\otimes} \hat{B}_{y}^{(0)}+h_{x} \hat{B}_{z}^{(0)}\right\} \\
& +C_{3} \exp \left[i\left(k_{g} z+\omega_{g} t\right)\right], \\
\tilde{B}_{x}^{(1)}= & \frac{i}{2} \frac{k_{g} z}{c}\left\{h_{x} \hat{E}_{x}^{(0)}-\left(h_{\oplus}+\frac{1}{2} h_{l}\right) \hat{E}_{y}^{(0)}+h_{y} \hat{E}_{z}^{(0)}\right\} \\
& -\frac{i}{2} k_{g} z\left\{\left(h_{b}-h_{\oplus}+h_{l}\right) \hat{B}_{x}^{(0)}+h_{\otimes} \hat{B}_{y}^{(0)}+h_{x} \hat{B}_{z}^{(0)}\right\} \\
& +C_{4} \exp \left[i\left(k_{g} z+\omega_{g} t\right)\right], \\
\text { and, } \tilde{E}_{z}^{(1)}= & h_{x} \hat{E}_{x}^{(0)}+h_{y} \hat{E}_{y}^{(0)}+\left(\frac{1}{2} h_{l}-h_{b}\right) \hat{E}_{z}^{(0)}, \\
\tilde{B}_{z}^{(1)}= & 0 .
\end{aligned}
$$

Equations (16) to (21) show that:

(1) If the background stable EM fields have all spatial components (i.e., the $x$-, $y$ - and $z$-components, here the HFGW propagates along the $z$-direction), then not only the EM response to the $\oplus$-type, $\otimes$-type polarization states in the GR framework can be produced, but also the EM response to the additional polarization states (the $x$-, $y$-, $b$ - and $l$-type polarization states) beyond the GR can be generated.

(2) The transverse polarization components $\left(\tilde{E}_{x}^{(1)}, \tilde{B}_{y}^{(1)}\right.$, $\left.\tilde{E}_{y}^{(1)}, \tilde{B}_{x}^{(1)}\right)$ of the perturbative EM fields show the spaceaccumulation effect (i.e., their strengths are proportional to the propagating distance $z$ of the HFGW in the background EM fields). This is because the HFGW (gravitons) and the perturbative EM waves (photons) have the same or almost the same propagating velocity (see Sect. 2). Thus, they can generate an optimal space-accumulation effect in the propagating direction, i.e., they and the results $[50,51]$ from the Feynman perturbative techniques and the Einstein-Maxwell equations are self-consistent. However, Eqs. (16) to (20) include new perturbative photon fluxes generated by the EM response to HFGWs in the longitudinal stable background EM fields $\hat{E}_{z}^{(0)}$ and $\hat{B}_{z}^{(0)}$ [see Eqs. (16) to (19)] and the longitudinal perturbative EM fields, Eq. (20). Moreover, even considering the 
correction to the propagating velocity for the HFGWs [45], such a correction to the space-accumulation effect can still be neglected even on a typical astronomical scale. Therefore, the final phase difference caused by the phase velocity $v_{P}$ does not cause any essential impact to the space-accumulation effect.

(3) Equations (16) to (19) also show that the perturbative EM fields propagating along the opposite direction of the HFGW [i.e., the perturbative EM fields containing the propagating factor $\left.\left(k_{g} z+\omega_{g} t\right)\right]$ do not have such a spaceaccumulation effect. Obviously, this is a self-consistent result to the EM perturbation, because there is no accumulation effect of energy for such EM fields in the opposite propagating direction of the HFGW.

(4) Unlike usual planar EM waves without longitudinal polarizations in free space, here the perturbative EM fields have the longitudinal polarizations, Eq. (20), and the longitudinal component is only from the EM response of the background electric fields, $\hat{E}_{x}^{(0)}, \hat{E}_{y}^{(0)}$ and $\hat{E}_{z}^{(0)}$ and not of the background magnetic fields $\hat{B}_{x}^{(0)}, \hat{B}_{y}^{(0)}$ and $\hat{B}_{z}^{(0)}$. Moreover, since there is a phase difference of $\pi / 2$ between the transverse perturbative EM fields $\left[\tilde{E}_{x}^{(1)}, \tilde{B}_{y}^{(1)}, \tilde{E}_{y}^{(1)}\right.$ and $\tilde{B}_{x}^{(1)}$, Eqs. (16) to (19)] and the longitudinal perturbative electric field $\tilde{E}_{z}^{(1)}$, Eq. (20), then the average values with respect to time of the transverse Poynting vector (i.e., the Poynting vector in the $x$ - and $y$-directions) are equal to zero [see Eq. (43)]. Clearly, such results ensure total momentum conservation in the interaction of the HFGW with the background EM fields. However, such a longitudinal perturbative electric field $\tilde{E}_{z}^{(1)}$ will play an important role in displaying and distinguishing the additional polarization states (the $x$-, $y$-, $b$ and $l$-polarizations) of the HFGWs in the 3DSR system (see below Sect. 4 and Appendix A).

2. The perturbative photon fluxes in the EM response.

Interaction of the HFGWs with the EM fields will generate perturbative EM power fluxes (signal EM power fluxes). In order to conveniently represent the above EM signal in the background noise photon flux fluctuation (see Appendix B), we will express them in quantum language, i.e., the perturbative photon fluxes (the PPFs, or signal photon fluxes). It is interesting to study the following cases:

(1) The EM response to the HFGWs in transverse background stable magnetic field $\hat{B}_{y}^{(0)}$.

In this case, $\hat{E}_{x}^{(0)}=\hat{E}_{y}^{(0)}=\hat{E}_{z}^{(0)}=\hat{B}_{x}^{(0)}=\hat{B}_{z}^{(0)}=0$ in Eqs. (16) to (20), i.e., only $\hat{B}_{y}^{(0)}$ has non-vanishing value. Then from Eqs. (16) to (19), we obtain the following results immediately:

$$
\begin{aligned}
\tilde{E}_{x}^{(1)} & =\frac{i}{2} k_{g} c z\left(h_{\oplus}+\frac{1}{2} h_{l}\right) \hat{B}_{y}^{(0)} \\
& =\frac{i}{2}\left(A_{\oplus}+\frac{\sqrt{2}}{2} A_{l}\right) \hat{B}_{y}^{(0)} k_{g} c z \exp \left[i\left(k_{g} z-\omega_{g} t\right)\right],
\end{aligned}
$$

$$
\begin{aligned}
\tilde{B}_{y}^{(1)} & =\frac{i}{2} k_{g} z\left(h_{\oplus}+\frac{1}{2} h_{l}\right) \hat{B}_{y}^{(0)} \\
& =\frac{i}{2}\left(A_{\oplus}+\frac{\sqrt{2}}{2} A_{l}\right) \hat{B}_{y}^{(0)} k_{g} z \exp \left[i\left(k_{g} z-\omega_{g} t\right)\right]
\end{aligned}
$$

and

$$
\begin{aligned}
\tilde{E}_{y}^{(1)} & =\frac{i}{2} k_{g} c z h_{\otimes} \hat{B}_{y}^{(0)}=\frac{i}{2} A_{\otimes} \hat{B}_{y}^{(0)} k_{g} c z \exp \left[i\left(k_{g} z-\omega_{g} t\right)\right] \\
\tilde{B}_{x}^{(1)} & =-\frac{i}{2} k_{g} z h_{\otimes} \hat{B}_{y}^{(0)} \\
& =-\frac{i}{2} A_{\otimes} \hat{B}_{y}^{(0)} k_{g} z \exp \left[i\left(k_{g} z-\omega_{g} t\right)\right] .
\end{aligned}
$$

Here, as well as in all discussions below, the perturbative EM fields propagating along the negative $z$-direction (i.e., the opposite propagating direction of the HFGWs) will be neglected, because they are very weak (they do not have the space-accumulation effect) or they are absent $[50,51]$. Therefore, we can make the constants $C_{1}, C_{2}, C_{3}$ and $C_{4}$ equal to zero in Eqs. (16) to (19).

The perturbative EM fields in Eqs. (22), (23) are similar to that in Refs. [50,51]. However, there is an important difference, i.e., the perturbative EM fields, Eq. (22), contain not only the contribution from the $\oplus$-type polarization state of the HFGWs, but also the contribution from the longitudinal polarization (the $l$-type polarization) of the HFGWs. In other words, the contribution of the $\oplus$-type polarization state is always accompanied by the $l$-type polarization states. Moreover, they have the same symbol in the amplitudes of the perturbative EM fields, Eq. (22), i.e., this is a constructive coherence effect between the $\oplus$-type polarization and the $l$-type polarization states. Thus, the interaction of the HFGWs having additional polarization states with the background transverse magnetic fields $\hat{B}_{y}^{(0)}$ will consume more energy [see Eq. (24)] than the HFGWs in the GR, i.e., the former will cause faster radiation damping than that of the latter for the HFGW sources in local regions.

Unlike such effect, the perturbative EM fields, Eq. (23), produced by the $\otimes$-type polarization state of the HFGWs, does not contain the contribution from the $l$-type or other additional polarization states.

From Eqs. (22), (23), the perturbative photon fluxes generated by the interaction of the HFGWs with the background magnetic field $\hat{B}_{y}^{(0)}$ can be given by (also see Fig. 3)

$$
\begin{aligned}
n_{z \oplus}^{(2)} & =\frac{1}{2 \mu_{0} \hbar \omega_{e}} \operatorname{Re}\left\langle\tilde{E}_{x}^{(1) *} \tilde{B}_{y}^{(1)}\right\rangle \\
& =\frac{1}{8 \mu_{0} \hbar \omega_{e}} k_{g}^{2} z^{2} c\left[\left(A_{\oplus}+\frac{\sqrt{2}}{2} A_{l}\right) \hat{B}_{y}^{(0)}\right]^{2}, \\
n_{z \bigotimes}^{(2)} & =\frac{1}{2 \mu_{0} \hbar \omega_{e}} \operatorname{Re}\left\langle\tilde{E}_{y}^{(1) *} \tilde{B}_{x}^{(1)}\right\rangle=\frac{k_{g}^{2} z^{2} c\left(A_{\otimes} \hat{B}_{y}^{(0)}\right)^{2}}{8 \mu_{0} \hbar \omega_{e}},
\end{aligned}
$$




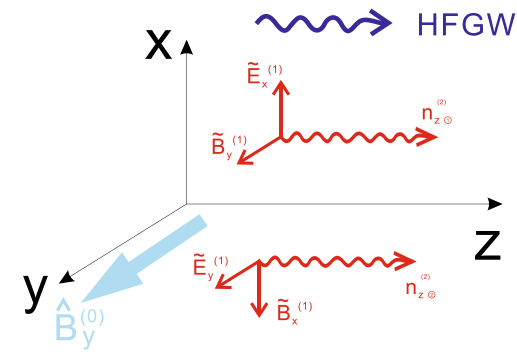

Fig. 3 When HFGW, Eq. (1), propagate in a transverse stable magnetic field $\tilde{B}_{y}^{(0)}$, the PPF $n_{z \text { (D) }}^{(2)}$, Eq. (24), is generated by $\tilde{E}_{x}^{(1)}$ and $\tilde{B}_{y}^{(1)}$, containing contributions from both $\oplus$-type and $l$-type polarizations, and the PPF $n_{z Z}^{(2)}$, Eq. (25), is produced by $\tilde{E}_{y}^{(1)}$ and $\tilde{B}_{x}^{(1)}$ containing only contribution from $\otimes$-type polarization

where * denotes the complex conjugate; the angular brackets represent the average over time, and the superscript " 2 " represents a second-order perturbation to the EM fields because they are proportional to the square of the HFGW amplitudes: $A_{\oplus}, A_{l}$ and $A_{\otimes}$. Here, $\omega_{e}=\omega_{g}$; there are two cases: (i) if the PPF is caused by the HFGWs interacting with the background stable galactic magnetic fields (see later part of this section), according to the electrodynamic equations in curved spacetime, the PPF has the same frequency to the HFGW which causes such PPF; (ii) in the 3DSR system (e.g. see Sect. 4), we need to adjust the frequencies of background EM waves (Gaussian beam) to match the HFGW frequencies, to meet the condition for the optimal EM response to HFGWs (i.e. the condition for resonance), and thus it is required that $\omega_{e}=\omega_{g}$.

(2) The EM response to the HFGW in transverse background stable magnetic field $\hat{B}_{x}^{(0)}$.

Then $\hat{E}_{x}^{(0)}=\hat{E}_{y}^{(0)}=\hat{E}_{z}^{(0)}=\hat{B}_{y}^{(0)}=\hat{B}_{z}^{(0)}=0$ in Eqs. (16) to (19), i.e., only $\hat{B}_{x}^{(0)}$ is not equal to zero. In the same way, from Eqs. (16) to (19), the corresponding perturbative photon fluxes are (see Fig. 4a)

$$
\begin{aligned}
n_{z \oplus}^{(2)} & =\frac{\operatorname{Re}\left\langle\tilde{E}_{x}^{(1) *} \tilde{B}_{y}^{(1)}\right\rangle}{2 \mu_{0} \hbar \omega_{e}}=\frac{k_{g}^{2} z^{2} c}{8 \mu_{0} \hbar \omega_{e}}\left(A_{\otimes} \hat{B}_{x}^{(0)}\right)^{2}, \\
n_{z \bigotimes}^{(2)} & =\frac{\operatorname{Re}\left\langle\tilde{E}_{y}^{(1) *} \tilde{B}_{x}^{(1)}\right\rangle}{2 \mu_{0} \hbar \omega_{e}} \\
& =\frac{k_{g}^{2} z^{2} c}{8 \mu_{0} \hbar \omega_{e}}\left[\left(A_{b}-A_{\oplus}+\sqrt{2} A_{l}\right) \hat{B}_{x}^{(0)}\right]^{2} .
\end{aligned}
$$

Equation (26) is very similar to Eq. (25), namely, the contribution of the $\otimes$-type polarization state is always independent of the additional polarization states. Meanwhile, Eqs. (24), (27) show that the contribution of the $\oplus$-type polarization state is always accompanied by the $l$-type, or the $b$-type and $l$-type polarization states.

Notice that although $\hat{B}_{y}^{(0)}$ and $\hat{B}_{x}^{(0)}$ are all the transverse stable magnetic fields, their EM response to the HFGW have certain differences. The term $n_{z \downarrow}^{(1)}$, Eq. (24), is a constructive coherence effect between the $\oplus$-type and the $l$-type polar- ization states (they have the same symbols), while $n_{z \bigotimes}^{(1)}$, Eq. (27), is a destructive coherence effect between the $\oplus$-type and the $b$-type, $l$-type polarization states (the $\oplus$-type and the $b$-type, $l$-type polarization states have the opposite symbols). This is because the impacts of the $b$-type polarization to the $\oplus$-type polarization in the $x x$-and the $y y$-components of the HFGW metric $h_{\mu v}$ are different [see Eq. (1)]. The former is "constructive superposition" (where the $b$-type and the $\oplus$-type polarizations have the same symbols), and the latter is "destructive superposition" (where the $b$-type and the $\oplus$-type polarizations have the opposite symbols). Moreover, the $l$-type polarization only appears in the $z z$-component of the metric $h_{\mu \nu}$. In this case, the EM response of $\hat{B}_{x}^{(0)}$ (the $y z$-component $F_{23}$ of the EM field tensor) and $\hat{B}_{y}^{(0)}$ (the $x z$ component $F_{13}$ of the EM field tensor) are non-symmetric. This is the physical origin of such a difference.

(3) The EM response to the HFGWs in the transverse background stable electric field $\hat{E}_{x}^{(0)}$ or $\hat{E}_{y}^{(0)}$.

In such cases (see Fig. $4 b, c$ ), the results are similar to the above cases, e.g.,

$$
\begin{aligned}
& n_{z \Phi}^{(2)}=\frac{\operatorname{Re}\left\langle\tilde{E}_{x}^{(1) *} \tilde{B}_{y}^{(1)}\right\rangle}{2 \mu_{0} \hbar \omega_{e}}=\frac{k_{g}^{2} z^{2}\left[\left(A_{\oplus}-\frac{\sqrt{2}}{2} A_{l}\right) \hat{E}_{x}^{(0)}\right]^{2}}{8 \mu_{0} c \hbar \omega_{e}}, \\
& n_{z \bigotimes}^{(2)}=\frac{\operatorname{Re}\left\langle\tilde{E}_{y}^{(1) *} \tilde{B}_{x}^{(1)}\right\rangle}{2 \mu_{0} \hbar \omega_{e}}=\frac{k_{g}^{2} z^{2}}{8 \mu_{0} c \hbar \omega_{e}}\left(A_{x} \hat{E}_{x}^{(0)}\right)^{2} .
\end{aligned}
$$

(4) The EM response to the HFGWs in the longitudinal stable EM fields $\hat{B}_{z}^{(0)}$ and $\hat{E}_{z}^{(0)}$.

In fact, whether according to the electrodynamic equations in curved spacetime [40,50], or the Feynman perturbation techniques to analyze the conversion of GWs into EM waves (and vice versa) [51], the GWs (including the HFGWs) in the GR framework (having only $\oplus$ - and $\otimes$-polarizations) do not generate any perturbation to the longitudinal static EM fields [40,50,51]. In contrast, the HFGWs having the additional polarization states will generate EM perturbations to such EM fields. Thus, their physical behaviors are quite different.

Putting $\hat{E}_{x}^{(0)}=\hat{E}_{y}^{(0)}=\hat{B}_{x}^{(0)}=\hat{B}_{y}^{(0)}=0$ in Eqs. (16) to (19), only $\hat{B}_{z}^{(0)}$ and $\hat{E}_{z}^{(0)}$ have non-vanishing values. Then Eqs. (16) to (19) are reduced to

$$
\begin{aligned}
\tilde{E}_{x}^{(1)} & =-\frac{i}{2} k_{g} z h_{x} \hat{E}_{z}^{(0)}-\frac{i}{2} k_{g} c z h_{y} \hat{B}_{z}^{(0)} \\
& =-\frac{i}{2} k_{g} z\left(A_{x} \hat{E}_{z}^{(0)}+c A_{y} \hat{B}_{z}^{(0)}\right) \exp \left[i\left(k_{g} z-\omega_{g} t\right)\right], \\
\tilde{B}_{y}^{(1)} & =-\frac{i}{2 c} k_{g} z h_{x} \hat{E}_{z}^{(0)}-\frac{i}{2} k_{g} z h_{y} \hat{B}_{z}^{(0)} \\
& =-\frac{i}{2} k_{g} z\left(\frac{1}{c} A_{x} \hat{E}_{z}^{(0)}+A_{y} \hat{B}_{z}^{(0)}\right) \exp \left[i\left(k_{g} z-\omega_{g} t\right)\right],
\end{aligned}
$$

$$
\tilde{E}_{y}^{(1)}=-\frac{i}{2} k_{g} z h_{y} \hat{E}_{z}^{(0)}+\frac{i}{2} k_{g} c z h_{x} \hat{B}_{z}^{(0)}
$$




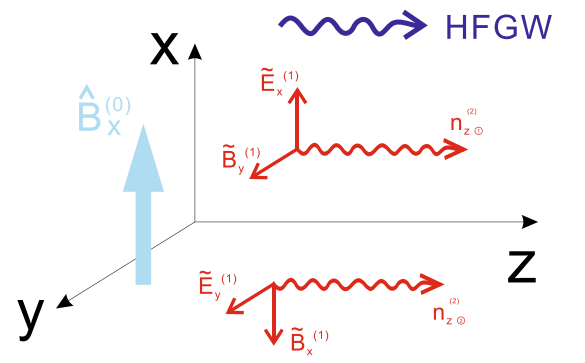

Fig. 4 Similar to Fig. 3, here: a $n_{z \infty}^{(2)}$, Eq. (26), caused only by $\otimes-$ polarization HFGWs; $n_{z Q}^{(2)}$, Eq. (27), caused by $\oplus$-, $b$ - and $l$-polarization HFGWs. b $n_{z \infty}^{(2)}$, Eq. (28), caused by $\oplus$ - and the $l$-polarization HFGWs;

$$
\begin{aligned}
& =-\frac{i}{2} k_{g} z\left(A_{y} \hat{E}_{z}^{(0)}-c A_{x} \hat{B}_{z}^{(0)}\right) \exp \left[i\left(k_{g} z-\omega_{g} t\right)\right] \\
\tilde{B}_{x}^{(1)} & =\frac{i}{2 c} k_{g} z h_{y} \hat{E}_{z}^{(0)}-\frac{i}{2} k_{g} z h_{x} \hat{B}_{z}^{(0)} \\
& =\frac{i}{2} k_{g} z\left(\frac{1}{c} A_{y} \hat{E}_{z}^{(0)}-A_{x} \hat{B}_{z}^{(0)}\right) \exp \left[i\left(k_{g} z-\omega_{g} t\right)\right]
\end{aligned}
$$

From Eqs. (30) to (33), the corresponding PPFs can be given by (see Fig. 4c)

$$
\begin{aligned}
& n_{z \Phi}^{(2)}=\frac{\operatorname{Re}\left\langle\tilde{E}_{x}^{(1) *} \tilde{B}_{y}^{(1)}\right\rangle}{2 \mu_{0} \hbar \omega_{e}}=\frac{k_{g}^{2} z^{2} c}{8 \mu_{0} \hbar \omega_{e}}\left(\frac{1}{c} A_{x} \hat{E}_{z}^{(0)}+A_{y} \hat{B}_{z}^{(0)}\right)^{2}, \\
& n_{z \bigotimes}^{(2)}=\frac{\operatorname{Re}\left\langle\tilde{E}_{y}^{(1) *} \tilde{B}_{x}^{(1)}\right\rangle}{2 \mu_{0} \hbar \omega_{e}}=\frac{k_{g}^{2} z^{2} c}{8 \mu_{0} \hbar \omega_{e}}\left(\frac{1}{c} A_{y} \hat{E}_{z}^{(0)}-A_{x} \hat{B}_{z}^{(0)}\right)^{2} .
\end{aligned}
$$

It is interesting to note that such PPFs are only produced by the pure additional polarization states (the $x$ - and $y$ polarizations, i.e., the vector mode gravitons). In other words, they are independent of the tensor-mode gravitons $(\oplus$ - and $\otimes$-polarizations) and the scalar-mode gravitons (the $b$-type and the $l$-type polarizations).

If we consider only the EM response of the longitudinal stable magnetic field $\hat{B}_{z}^{(0)}$, i.e., $\hat{E}_{z}^{(0)}=0$ in Eqs. (34) and (35), then the equations are reduced to

$$
\begin{aligned}
& n_{z \Phi}^{(2)}=\frac{k_{g}^{2} z^{2} c}{8 \mu_{0} \hbar \omega_{e}}\left(A_{y} \hat{B}_{z}^{(0)}\right)^{2}, \\
& n_{z \bigotimes}^{(2)}=\frac{k_{g}^{2} z^{2} c}{8 \mu_{0} \hbar \omega_{e}}\left(A_{x} \hat{B}_{z}^{(0)}\right)^{2} .
\end{aligned}
$$

In this case, the $x$-type and the $y$-type polarizations of the HFGWs can be more clearly displayed and have obvious physical effects. In fact, according to contemporary astronomic observation [52], it is certain that there are very widespread background galactic-extragalactic stable magnetic fields with strengths $\sim 10^{-11} \mathrm{~T}$ to $10^{-9} \mathrm{~T}$ within $1 \mathrm{Mpc}$ in galaxies and galaxy clusters (see Fig. 5). These magnetic fields might provide a large space-accumulation effect during the propagating of the HFGWs from possible sources to the Earth. This means that either the EM response to HFGWs in the transverse background EM fields [see Eqs. (24), (25), (26), (27), (28), (29)] or in the longitudinal background EM fields [see Eqs. (34) to (37)], it is all possible to detect or observe such an effect provided these background EM fields are distributed in a very wide region. Since the wide distribution of the background galactic-extragalactic magnetic fields has been observed [52], the EM response to the HFGWs in such background magnetic fields would have more realistic significance than that in the background electric fields, and the EM response in the background longitudinal magnetic fields, Eqs. (36) and (37), might provide observational evidence produced by the pure additional polarization states (the $x$ - and the $y$-polarizations of the HFGW).

Here, we can present a brief estimation of the perturbative photon fluxes (PPFs) in the EM response of galacticextragalactic background stable EM fields to the HFGWs. In the above discussion, we assume that the GWs are planar waves. For the observers and detection systems in the far-field region from the GW sources, such an assumption is obviously reasonable. However, if we study a large space-accumulation effect in the EM response to the HFGWs, which concerns a large distance from their sources to the Earth, then such HFGWs should be considered as the spherical GWs for GW sources in the local regions, i.e., these HFGW sources would be in approximately a point-like distribution. This means that the amplitudes of the HFGWs emitted by such sources would be inversely proportional to the propagating distance $z$ (i.e., along the $z$-axis in our coordinate system; see Ref. [45]). Then the amplitudes $A_{\oplus}, A_{\otimes}, A_{x}, A_{y}, A_{b}$ and $A_{l}$ of the HFGWs in Eqs. (24), (25), (26), (27), (28), (29), and (34) to (37) should be replaced by $A_{\oplus} z_{d} / z, A_{\otimes} z_{d} / z, A_{x} z_{d} / z$, $A_{y} z_{d} / z, A_{b} z_{d} / z$ and $A_{l} z_{d} / z$, respectively, where $z_{d}$ is the distance between the GW source to the Earth, and the $A_{\oplus}$, $A_{\otimes}, A_{x}, A_{y}, A_{b}$ and $A_{l}$ are the amplitudes around the Earth. 
Then approximately the PPFs generated by the EM response in the background stable magnetic fields can be reduced to the following forms, respectively:

$$
\begin{aligned}
& n_{\oplus, l}^{(2)}=\frac{k_{g}^{2} z_{d}^{2} c}{8 \mu_{0} \hbar \omega_{e}}\left[\left(A_{\oplus}+\frac{\sqrt{2}}{2} A_{l}\right) \hat{B}_{y}^{(0)}\right]^{2}, \\
& n_{\otimes}^{(2)}=\frac{k_{g}^{2} z_{d}^{2} c}{8 \mu_{0} \hbar \omega_{e}}\left[\left(A_{\otimes} \hat{B}_{x}^{(0)}\right)^{2}+\left(A_{\otimes} \hat{B}_{y}^{(0)}\right)^{2}\right], \\
& n_{\oplus, b, l}^{(2)}=\frac{k_{g}^{2} z_{d}^{2} c}{8 \mu_{0} \hbar \omega_{e}}\left[\left(A_{b}-A_{\oplus}+\sqrt{2} A_{l}\right) \hat{B}_{x}^{(0)}\right]^{2}, \\
& n_{x}^{(2)}=\frac{k_{g}^{2} z_{d}^{2} c}{8 \mu_{0} \hbar \omega_{e}}\left(A_{x} \hat{B}_{z}^{(0)}\right)^{2}, \\
& n_{y}^{(2)}=\frac{k_{g}^{2} z_{d}^{2} c}{8 \mu_{0} \hbar \omega_{e}}\left(A_{y} \hat{B}_{z}^{(0)}\right)^{2} .
\end{aligned}
$$

The $A_{\oplus}, A_{\otimes}, A_{x}, A_{y}, A_{b}$ and $A_{l}$ are amplitudes in the far-field region (e.g., the Earth). As mentioned above, this is because the large space-accumulation effect would compensate for the decay of the spherical HFGWs and their weakness of the far-field amplitudes.

Fortunately, related observation shows that the distribution region of the stable background magnetic field (see Fig. 5) in our galaxy is at nearly a few thousand light-year distance $\left(\sim 10^{19} \mathrm{~m}[52,53]\right)$, and such a magnetic field basically keeps fixed direction and intensity, $\sim 10^{-9}$ to $10^{-10}$ Tesla; thus this background magnetic field would provide an effective space-accumulation effect to the PPFs produced by the HFGWs.

According to a related estimation for the distance of possible HFGW sources of the braneworld scenarios [see, e.g., Ref. [21]] in our galaxy, they may be $\sim 10^{18} \mathrm{~m}$ to $\sim 10^{19} \mathrm{~m}$ away from the Earth. In this case, if the amplitudes of the HFGWs $\left(v \sim 10^{9}\right.$ to $\left.10^{14} \mathrm{~Hz}\right)$ can reach up to the predicted values once they arrive at Earth, i.e., $h \sim 10^{-25}$ (lower bound) to $h \sim 10^{-21}$ (upper bound). Then the related signal photon flux densities could reach up to the value in Table 1.

Table 1 shows estimations of the signal photon flux densities generated by the EM response of the background stable magnetic fields to the HFGWs. Here $\hat{B}^{(0)} \sim 10^{-11} T$ to $\sim 10^{-10} \mathrm{~T}$; the propagating distance $\Delta z \sim 10^{19} \mathrm{~m}$ of the HFGWs from the source to the Earth; the amplitudes of the HFGWs at the Earth are $h \sim 10^{-21}$ to $10^{-25}$ [21].

Obviously, the PPFs (the EM signals) in Table 1 are larger than the minimal detectable EM power $\sim 10^{-22}$ to $\sim 10^{-24} \mathrm{Wm}^{-2}$ under current technological condition [e.g., the Five-hundred-meter Aperture Spherical Telescope (FAST) [54,55], which has been constructed in 2017 in Guizhou province of China. However, the above EM signals and the space background EM noise often accompany each other. Thus distinguishing of the EM signals from the background EM noise at the Earth will be a major challenge. For-

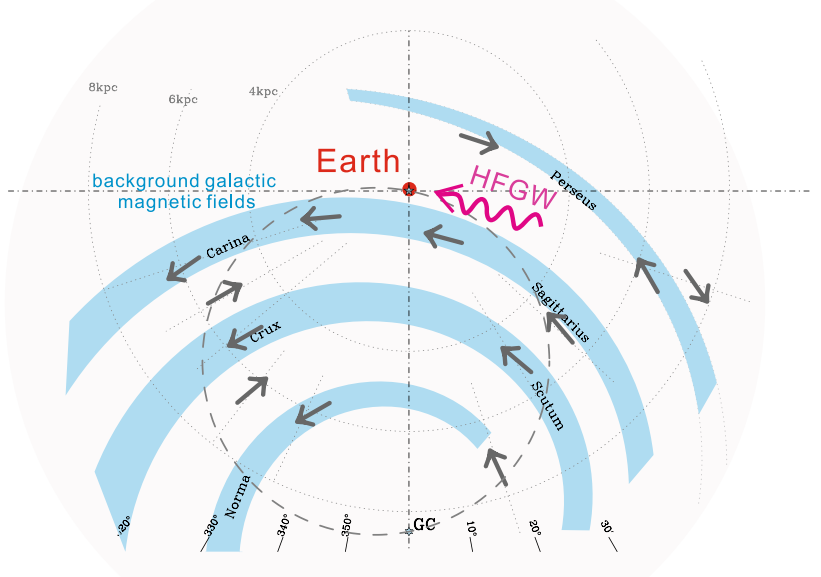

Fig. 5 The spatial scale of distribution for the background magnetic fields in the Milky Way reaches up to $\sim$ thousand light-years long [52, $53,56]$, and the magnetic fields have a stable strength and direction in the scale. Thus any HFGWs passing through the background magnetic field would generate a significant space-accumulation effect in the Earth region. This figure is made based on some materials of Refs. [56]

tunately, the PPFs (the EM signals) produced by the HFGWs from the braneworld also contain important characteristics of the HFGWs, such as the discrete spectrum property, special waveform, strength distribution and very-high frequency features. Thus, in principle, it is always possible to distinguish and display the PPFs (the EM signals) from the background EM noise.

It should be pointed out again that, if the propagating direction of the GWs (including the HFGWs) and the pointing direction of the background EM fields are parallel to each other (i.e., only the longitudinal stable EM fields $\hat{E}_{z}^{(0)}, \hat{B}_{z}^{(0)}$ exist here), then GWs in the GR framework do not generate any perturbative effect to the EM fields [50,51]. In comparison, the GWs having additional polarization states will generate perturbative EM fields to the background EM fields in any case, even if the pointing direction of the background EM fields and the propagating direction of the HFGWs are parallel to each other [see Eqs. (34), (35), (41), (42)], which are just the perturbation effects produced by the pure additional polarization states ( $x$ - and $y$-polarizations, i.e., vector mode gravitons) of the HFGWs.

In this case, the displaying condition of the HFGWs can be greatly relaxed. In other words, this is an important symbol to display and distinguish the HFGWs in the GR framework and the HFGWs beyond the GR.

It should be pointed out that the coupling between the longitudinal perturbative electric fields $\tilde{E}_{z}^{(1)}$, Eq. (20), and the transverse perturbative magnetic fields, Eqs. (17) and (19), 
Table 1 The EM response to the KK-HFGWs from the braneworld [21] in the galactic-extragalactic magnetic fields, where $N^{(2)}$ is the perturbative photon flux densities $\left(\mathrm{m}^{-2} \mathrm{~s}^{-1}\right), P$ is corresponding EM signal power flux densities $\left(\mathrm{Wm}^{-2}\right)$ at the Earth. Here, " $A$ " represents possible GW amplitude around the Earth [could be one of $A_{\oplus}, A_{\otimes}, A_{x}$, $A_{y}, A_{b}, A_{l}$ in Eqs. (38) to (42)]

\begin{tabular}{lll}
\hline Background magnetic field $\hat{B}^{(0)}$ & $v_{g} \sim 10^{9} \mathrm{~Hz}$ & $v_{g} \sim 10^{12} \mathrm{~Hz}$ \\
\hline$\hat{B}^{(0)}=10^{-10} T$ & & \\
$A \sim 10^{-25}$ & $N^{(2)}=6 \times 10^{4}\left(\mathrm{~m}^{-2} s^{-1}\right)\left(P=10^{-19} \mathrm{Wm}^{-2}\right)$ & $N^{(2)}=6 \times 10^{7}\left(\mathrm{~m}^{-2} s^{-1}\right)\left(P=10^{-13} \mathrm{Wm}^{-2}\right)$ \\
$A \sim 10^{-23}$ & $N^{(2)}=6 \times 10^{8}\left(\mathrm{~m}^{-2} s^{-1}\right)\left(P=10^{-15} \mathrm{Wm}^{-2}\right)$ & $N^{(2)}=6 \times 10^{11}\left(\mathrm{~m}^{-2} s^{-1}\right)\left(P=10^{-9} \mathrm{Wm}^{-2}\right)$ \\
$A \sim 10^{-21}$ & $N^{(2)}=6 \times 10^{12}\left(\mathrm{~m}^{-2} s^{-1}\right)\left(P=10^{-11} \mathrm{Wm}^{-2}\right)$ & $N^{(2)}=6 \times 10^{15}\left(\mathrm{~m}^{-2} s^{-1}\right)\left(P=10^{-5} \mathrm{Wm}^{-2}\right)$ \\
$\hat{B}^{(0)}=10^{-11} T$ & & \\
$A \sim 10^{-25}$ & $N^{(2)}=6 \times 10^{2}\left(\mathrm{~m}^{-2} s^{-1}\right)\left(P=10^{-21} \mathrm{Wm}^{-2}\right)$ & $N^{(2)}=6 \times 10^{5}\left(\mathrm{~m}^{-2} s^{-1}\right)\left(P=10^{-15} \mathrm{Wm}^{-2}\right)$ \\
$A \sim 10^{-23}$ & $N^{(2)}=6 \times 10^{6}\left(\mathrm{~m}^{-2} s^{-1}\right)\left(P=10^{-17} \mathrm{Wm}^{-2}\right)$ & $N^{(2)}=6 \times 10^{9}\left(\mathrm{~m}^{-2} s^{-1}\right)\left(P=10^{-11} \mathrm{Wm}^{-2}\right)$ \\
$A \sim 10^{-21}$ & $N^{(2)}=6 \times 10^{10}\left(\mathrm{~m}^{-2} s^{-1}\right)\left(P=10^{-13} \mathrm{Wm}^{-2}\right)$ & $N^{(2)}=6 \times 10^{13}\left(\mathrm{~m}^{-2} s^{-1}\right)\left(P=10^{-7} \mathrm{Wm}^{-2}\right)$ \\
\hline
\end{tabular}

do not generate any transverse PPFs, i.e.,

$$
\begin{aligned}
& n_{y}^{(2)}=\frac{\operatorname{Re}\left\langle\tilde{E}_{z}^{(1) *} \tilde{B}_{x}^{(1)}\right\rangle}{2 \mu_{0} \hbar \omega_{e}}=0, \\
& n_{x}^{(2)}=\frac{\operatorname{Re}\left\langle\tilde{E}_{z}^{(1) *} \tilde{B}_{y}^{(1)}\right\rangle}{2 \mu_{0} \hbar \omega_{e}}=0 .
\end{aligned}
$$

This is because $\tilde{E}_{z}^{(1)}$, Eq. (20), and $\tilde{B}_{x}^{(1)}, \tilde{B}_{y}^{(1)}$, Eqs. (17), (19), have a phase difference of $\pi / 2$. Therefore, the average values of the transverse perturbative EM power fluxes, Eq. (43), are vanishing. In fact, such results ensure the total momentum conservation in the interaction of the HFGWs (gravitons) with the background EM fields. However, the longitudinal perturbative electric field $\tilde{E}_{z}^{(1)}$ will play an important role in the 3DSR system (see Sect. 4). We will show that the transverse PPFs generated by the coupling between the $\tilde{E}_{z}^{(1)}$ and the transverse magnetic fields of the Gaussian beam (GB) might display the effect of the pure additional polarization states (the $x$-type, $y$-type, $l$-type and $b$-type polarizations) of the HFGWs.

Nevertheless, if we only consider the interaction of the HFGWs with the galactic-extragalactic background magnetic fields, and only use some large-scaled facilities (e.g. the FAST) to observe the PPFs produced by such interaction, we cannot separate all possible six polarization states of the HFGWs, due to the FAST being fixed on the Earth (and we cannot rotate it as a whole in large ranges of the polar and azimuth angles just like rotating the 3DSR), so we only record the averaged effect from such PPFs, which contain information of the combined HFGW polarizations (including possible extra-polarizations). Therefore, we need to consider the coincident experiments of the galactic-extragalactic cases (e.g. the FAST observation) and the 3DSR. The FAST would provide a supporting method to identify the potential HFGW signals, and the 3DSR is possible to obtain the better way and ability to separate all possible six polarizations, e.g., it would observe and separate the PPFs caused by the pure $\otimes-, x-$ and y-polarizations, and the contributions of the pure $\oplus$-, $b$ - and $l$-polarizations can also be straightforwardly calculated based on the observed signals (see Sect. 4).

\section{EM response to the HFGWs having additional polarization states in the $3 \mathrm{D}$-EM synchro-resonance system (3DSR system)}

The proposed 3DSR system consists of the background Gaussian-type photon flux [Gaussian beam (GB)] and the background static EM fields, $\hat{E}^{(0)}$ and $\hat{B}^{(0)}$ (see Figs. 1 and 2 ). The 3DSR was discussed in Refs. [40,41], so we shall not repeat it in detail here and we only present a brief description. The 3DSR is proposed as a HFGW detection system based on the effect of EM response to GWs. The interaction of HFGWs with the background static EM fields in the 3DSR will generate the second order signal perturbative EM waves (inverse Gertsenshtein effect), and such signal EM waves will be amplified by the effect of resonance with the Gaussian beam in the 3DSR, and thus lead to the first order signal perturbative EM waves. We use the cutting edge methods of single photon detections to measure these first order EM signals, in order to find if the HFGWs come into the detection system. In this article, the 3DSR system is different from that in previous studies, and we update it into a new system for probing the HFGWs having additional polarizations to display related novel effects, i.e.:

(1) Unlike previous EM detection schemes, here the 3DSR system contains not only the static magnetic field, but also the static electric field, and their directions can be adjusted. In this special coupling between the static EM fields and the Gaussian-type photon flux, the perturbative EM signals generated by the different polarization states (the tensor-, the vector- and the scalar-mode gravitons in the high-frequency band), in principle, can be effectively distinguished and displayed. 
(2) The EM signals generated by the interaction of the HFGWs with the background static EM fields will have the same frequencies with the HFGWs. Thus once the GB is adjusted to the resonance frequency band for the HFGWs, then the first-order perturbative EM power fluxes [the perturbative photon fluxes (PPFs), i.e., the signal photon fluxes] also have such frequency band. This means that the 3DSR can be a detection system of a broad frequency band.

In order to make the system having a good sensitivity to distinguish the PPFs generated by the different polarization states of the HFGWs, we select a new group of wave beam solutions for the GB in the framework of the quantum electronics (also see Appendix A):

$$
\begin{aligned}
\tilde{E}_{x}^{(0)}= & \psi_{e x}=\psi=\frac{\psi_{0}}{\sqrt{1+(z / f)^{2}}} \cdot \\
& \exp \left(\frac{-r^{2}}{W^{2}}\right) \exp \left\{i \left[\left(k_{e} z-\omega_{e} t\right)-\tan ^{-1} \frac{z}{f}\right.\right. \\
& \left.\left.+\frac{k_{e} r^{2}}{2 R}+\delta\right]\right\}, \\
\tilde{E}_{y}^{(0)}= & \psi_{e y}=0, \\
\tilde{E}_{z}^{(0)}= & \psi_{e z}=2 x F_{1}\left(\mathbf{x}, k_{e}, W\right)=2 r \cos \phi F_{1}\left(\mathbf{x}, k_{e}, W\right),(44) \\
\tilde{B}_{x}^{(0)}= & \psi_{b x}=-\frac{i}{\omega_{e}} \frac{\partial \psi_{e z}}{\partial y}=\frac{\sin 2 \phi}{\omega_{e}} F_{2}\left(\mathbf{x}, k_{e}, W\right), \\
\tilde{B}_{y}^{(0)}= & \psi_{b y}=-\frac{i}{\omega_{e}}\left(\frac{\partial \psi_{e x}}{\partial z}-\frac{\partial \psi_{e z}}{\partial x}\right) \\
= & \frac{1}{\omega_{e}}\left[F_{4}\left(\mathbf{x}, k_{e}, W\right)-i\left(2 F_{1}\left(\mathbf{x}, k_{e}, W\right)\right.\right. \\
& \left.\left.+\cos ^{2} \phi F_{3}\left(\mathbf{x}, k_{e}, W\right)\right)\right], \\
\tilde{B}_{z}^{(0)}= & \frac{i}{\omega_{e}} \frac{\partial \psi_{e x}}{\partial y}=\frac{-\sin \phi}{\omega_{e}}\left[\frac{k_{e} r}{R}+\frac{i 2 r}{W_{0}^{2}\left[1+(z / f)^{2}\right]}\right] \psi,
\end{aligned}
$$

where $\tilde{E}_{x}^{(0)}, \tilde{E}_{y}^{(0)}, \tilde{E}_{z}^{(0)}, \tilde{B}_{x}^{(0)}, \tilde{B}_{y}^{(0)}$ and $\tilde{B}_{z}^{(0)}$ are the electric and magnetic components in a Cartesian coordinate system for the GB, respectively, and only the x-component $\tilde{E}_{x}^{(0)}$ of the electric field has a standard form of circular mode of the fundamental frequency GB [57]. The concrete expressions of functions $F_{1}, F_{2}, F_{3}$ and $F_{4}$ can be found in Appendix A.

In fact, there are different solutions of the wave beam for the Helmholtz equation, and they can be the Gaussian-type wave beams or the quasi-Gaussian-type wave beams. One of the reasons of selecting such wave beam solutions, Eqs. (44) and (45), is that we will have an optimal coupling between the Gaussian-type photon flux and the background static EM fields, and this will make the PPFs (the signal photon fluxes) and the background noise photon fluxes show very different physical behaviors in the special local region. These physical behaviors include the propagating direction, strength distribution, decay rate, wave impedance, etc. (see the text below and Appendix B). Thus, such results will greatly improve the distinguishability between the signal photon fluxes and the background noise photons. Also, they will greatly increase the separability among the tensor-mode, the vector-mode and the scalar-mode gravitons. This is the physical origin of the very low standard quantum limit of the 3DSR system (i.e., the high sensitivity of the 3DSR system; see, e.g., Ref. [58]).

By using Eqs. (44) and (45), the average values of the transverse background photon flux (the Gaussian-type photon flux) with respect to time in cylindrical polar coordinates can be given by

$$
\begin{aligned}
n_{\phi}^{(0)}= & -n_{x}^{(0)} \sin \phi+n_{y}^{(0)} \cos \phi \\
= & -\frac{c}{\hbar \omega_{e}}\left\langle T^{(0)}\right\rangle \sin \phi+\frac{c}{\hbar \omega_{e}}\left\langle T^{(0)}\right\rangle \cos \phi \\
= & \frac{-1}{2 \mu_{0} \hbar \omega_{e}} \operatorname{Re}\left\langle\psi_{e z}^{*} \psi_{b y}\right\rangle \sin \phi \\
& +\frac{1}{2 \mu_{0} \hbar \omega_{e}} \operatorname{Re}\left\langle\psi_{e x}^{*} \psi_{b z}\right\rangle \cos \phi \\
& +\frac{1}{2 \mu_{0} \hbar \omega_{e}} \operatorname{Re}\left\langle\psi_{e z}^{*} \psi_{b x}\right\rangle \cos \phi \\
= & f_{\phi}^{(0)} \exp \left(-\frac{2 r^{2}}{W^{2}}\right) \sin 2 \phi,
\end{aligned}
$$

where $T^{01}$ and $T^{02}$ are 01 - and 02-components of the energymomentum tensor for the background EM wave (the GB), and [57]

$\left.n_{\phi}^{(0)}\right|_{x=0}=\left.n_{\phi}^{(0)}\right|_{y=0}=0$,

i.e., the transverse background photon flux at the longitudinal symmetry surface (the $y z$-plane and the $x z$-plane) of the GB is equal to zero (see Fig. 7). In fact, this is the necessary condition for the stability of GB.

From Eqs. (44), (45) and (16) to (20), and under the resonance condition $\left(\omega_{e}=\omega_{g}\right)$, the transverse perturbative photon fluxes (PPFs) in cylindrical polar coordinates can be given by

$$
\begin{aligned}
n_{\phi}^{(1)}= & -n_{x}^{(1)} \sin \phi+n_{y}^{(1)} \cos \phi=-\frac{c}{2 \hbar \omega_{e}} \\
& \cdot\left\langle T^{01}\right\rangle_{\omega_{e}=\omega_{g}} \sin \phi+\frac{c}{2 \hbar \omega_{e}}\left\langle T^{02}\right\rangle_{\omega_{e}=\omega_{g}} \cos \phi \\
= & -\frac{1}{2 \mu_{0} \hbar \omega_{e}} \operatorname{Re}\left\langle\tilde{E}_{y}^{(1) *} \tilde{B}_{z}^{(0)}\right\rangle_{\omega_{e}=\omega_{g}} \sin \phi \\
& +\frac{1}{2 \mu_{0} \hbar \omega_{e}} \operatorname{Re}\left\langle\tilde{E}_{z}^{(1) *} \tilde{B}_{x}^{(0)}\right\rangle_{\omega_{e}=\omega_{g}} \cos \phi \\
& -\frac{1}{2 \mu_{0} \hbar \omega_{e}} \operatorname{Re}\left\langle\tilde{E}_{x}^{(1) *} \tilde{B}_{z}^{(0)}\right\rangle_{\omega_{e}=\omega_{g}} \cos \phi \\
& +\frac{1}{2 \mu_{0} \hbar \omega_{e}} \operatorname{Re}\left\langle\tilde{E}_{z}^{(1) *} \tilde{B}_{x}^{(0)}\right\rangle_{\omega_{e}=\omega_{g}} \cos \phi
\end{aligned}
$$


where $\left\langle T^{01}\right\rangle_{\omega_{e}=\omega_{g}}$ and $\left\langle T^{02}\right\rangle_{\omega_{e}=\omega_{g}}$ are average values with respect to time of 01 - and 02-components of the energymomentum tensor for the first-order perturbative EM fields.

In the following, we shall study the EM response to the HFGWs with the additional polarization states in some typical cases.

1. EM response to the HFGWs in the coupling system between the transverse background static magnetic field $\hat{B}_{y}^{(0)}$ and the GB.

Then $\hat{E}_{x}^{(0)}=\hat{E}_{y}^{(0)}=\hat{E}_{z}^{(0)}=\hat{B}_{x}^{(0)}=\hat{B}_{z}^{(0)}=0$ and only $\hat{B}_{y}^{(0)} \neq 0$. In fact, this is a coupling system between the transverse static magnetic fields $\tilde{B}_{y}^{(0)}$ and the GB. In this case, from Eqs. (22), (23), (44), (45) and (48), the concrete forms of the transverse PPFs can be obtained:

$$
\begin{aligned}
& \text { let }\left[\frac{k_{e} r^{2}}{2 R}-\tan ^{-1}\left(\frac{z}{f}\right)+\delta\right]=\mathscr{P}, \\
& \text { let } \frac{\exp \left(-\frac{r^{2}}{W^{2}}\right) k_{g}}{\left[1+(z / f)^{2}\right]^{\frac{1}{2}}\left(z+f^{2} / z\right)}=\mathscr{A}, \\
& \text { let } \frac{\exp \left(-\frac{r^{2}}{W^{2}}\right)}{W_{0}^{2}\left[1+(z / f)^{2}\right]^{\frac{3}{2}}}=\mathscr{B},
\end{aligned}
$$

and in the same way

$$
\begin{aligned}
n_{\phi-\otimes}^{(1)}= & \frac{-1}{2 \mu_{0} \hbar \omega_{e}} \operatorname{Re}\left\langle\tilde{E}_{y}^{(1) *} \tilde{B}_{z}^{(0)}\right\rangle_{\omega_{e}=\omega_{g}} \sin \phi \\
= & \frac{A_{\otimes} \hat{B}_{y}^{(0)} \psi_{0} \Delta z r}{\mu_{0} \hbar \omega_{e}}\left\{\frac{\mathscr{A}}{2} \sin \mathscr{P}+\mathscr{B} \cos \mathscr{P}\right\} \sin ^{2} \phi \\
n_{\phi-\oplus, l}^{(1)}= & \frac{1}{2 \mu_{0} \hbar \omega_{e}} \operatorname{Re}\left\langle\tilde{E}_{x}^{(1) *} \tilde{B}_{z}^{(0)}\right\rangle_{\omega_{e}=\omega_{g}} \cos \phi \\
= & \frac{\left(A_{\oplus}+\frac{\sqrt{2}}{2} A_{l}\right) \hat{B}_{y}^{(0)} \psi_{0} \Delta z r}{\mu_{0} \hbar \omega_{e}}\left(\frac{\mathscr{A}}{4} \sin \mathscr{P}\right. \\
& \left.+\frac{\mathscr{B}}{2} \cos \mathscr{P}\right) \sin 2 \phi,
\end{aligned}
$$

where $\Delta z$ is the spatial scale of the transverse static magnetic field $\hat{B}_{y}^{(0)}$ in the 3 DSR system. Equation (50) shows that the transverse PPF $n_{\phi-\otimes}^{(1)}$ is produced by the pure $\otimes$-type polarization of HFGWs (this means turning back to the case with only tensorial mode and only background magnetic fields, i.e., the case studied by previous work $[40,42]$ ), while Eq. (51) represents the transverse $\operatorname{PPF} n_{\phi-\oplus, l}^{(1)}$ generated by the combination state of the $\oplus$-type and the $l$-type polarizations of the HFGWs.

2. EM response to the HFGWs in the coupling system between the transverse background static magnetic field $\hat{B}_{x}^{(0)}$ and the GB.

Then $\hat{E}_{x}^{(0)}=\hat{E}_{y}^{(0)}=\hat{E}_{z}^{(0)}=\hat{B}_{y}^{(0)}=\hat{B}_{z}^{(0)}=0$ and only $\hat{B}_{x}^{(0)} \neq 0$. In this case, from Eqs. (45) and (48), we have

$n_{\phi-\oplus, b, l}^{(1)}=\frac{-1}{\mu_{0} \hbar \omega_{e}} \operatorname{Re}\left\langle\tilde{E}_{y}^{(1) *} \tilde{B}_{z}^{(0)}\right\rangle_{\omega_{e}=\omega_{g}} \sin \phi$

$$
\begin{aligned}
= & \frac{\left(A_{b}-A_{\oplus}+\sqrt{2} A_{l}\right) \hat{B}_{x}^{(0)} \psi_{0} \Delta z r}{\mu_{0} \hbar \omega_{e}} \\
& \cdot\left(\frac{\mathscr{A}}{2} \sin \mathscr{P}+\mathscr{B} \cos \mathscr{P}\right) \sin ^{2} \phi \\
n_{\phi-\otimes}^{(1)}= & \frac{1}{\mu_{0} \hbar \omega_{e}} \operatorname{Re}\left\langle\tilde{E}_{x}^{(1) *} \tilde{B}_{z}^{(0)}\right\rangle_{\omega_{e}=\omega_{g}} \cos \phi \\
= & \frac{A_{\otimes} \hat{B}_{x}^{(0)} \psi_{0} k_{g} \Delta z r}{\mu_{0} \hbar \omega_{e}}\left\{\frac{\mathscr{A}}{4} \sin \mathscr{P}+\frac{\mathscr{B}}{2} \cos \mathscr{P}\right\} \sin 2 \phi .
\end{aligned}
$$

Equation (52) shows that the $\operatorname{PPF} n_{\phi-\oplus, b, l}^{(1)}$ is produced by the combination state of the $\oplus$-type, $b$-type and $l$-type polarizations of the HFGWs, while Eq. (53) represents the fact that the $\operatorname{PPF} n_{\phi-\otimes}^{(1)}$ is generated by the pure $\otimes$-type polarization of the HFGWs (this also boils down to the case with only tensorial mode and magnetic fields, which was studied in previous work $[40,42])$.

3. The EM response to the HFGWs in the coupling system between the longitudinal background static magnetic field $\hat{B}_{z}^{(0)}$ and the GB.

Then $\hat{E}_{x}^{(0)}=\hat{E}_{y}^{(0)}=\hat{E}_{z}^{(0)}=\hat{B}_{x}^{(0)}=\hat{B}_{y}^{(0)}=0$ and only $\hat{B}_{z}^{(0)} \neq 0$. In this case, under the resonance condition ( $\left.\omega_{e}=\omega_{g}\right)$, from Eqs. (44), (45) and (48), the concrete forms of the transverse PPFs can be given by

$$
\begin{aligned}
n_{\phi-x}^{(1)} & =\frac{-1}{\mu_{0} \hbar \omega_{e}} \operatorname{Re}\left\langle\tilde{E}_{y}^{(1) *} \tilde{B}_{z}^{(0)}\right\rangle_{\omega_{e}=\omega_{g}} \sin \phi \\
& =\frac{A_{x} \hat{B}_{z}^{(0)} \psi_{0} \Delta z r}{\mu_{0} \hbar \omega_{e}}\left\{\frac{\mathscr{A}}{2} \sin \mathscr{P}+\mathscr{B} \cos \mathscr{P}\right\} \sin ^{2} \phi \\
n_{\phi-y}^{(1)} & =\frac{1}{\mu_{0} \hbar \omega_{e}} \operatorname{Re}\left\langle\tilde{E}_{x}^{(1) *} \tilde{B}_{z}^{(0)}\right\rangle_{\omega_{e}=\omega_{g}} \cos \phi \\
& =\frac{A_{y} \hat{B}_{z}^{(0)} \psi_{0} \Delta z r}{\mu_{0} \hbar \omega_{e}}\left\{\frac{\mathscr{A}}{4} \sin \mathscr{P}+\frac{\mathscr{B}}{2} \cos \mathscr{P}\right\} \sin 2 \phi
\end{aligned}
$$

Eqs. (54) and (55) show that the transverse PPFs $n_{\phi-x}^{(1)}$ and $n_{\phi-y}^{(1)}$ are generated by the pure $x$-type and the pure $y$-type polarizations of the HFGWs, respectively.

4. The EM response to the HFGWs in the coupling system between the transverse background static electric field $\hat{E}_{x}^{(0)}$ and the GB.

Then $\hat{E}_{y}^{(0)}=\hat{E}_{z}^{(0)}=\hat{B}_{x}^{(0)}=\hat{B}_{y}^{(0)}=\hat{B}_{z}^{(0)}=0$ and only $\hat{E}_{x}^{(0)} \neq 0$. In this case from Eqs. (20), (45) and (48), in the same way, under the resonance condition $\left(\omega_{e}=\omega_{g}\right)$, the transverse PPFs can be given by

$$
\begin{aligned}
n_{\phi-\oplus, l}^{(1)} & =\frac{-1}{2 \mu_{0} \hbar \omega_{e}} \operatorname{Re}\left\langle\tilde{E}_{x}^{(1) *} \tilde{B}_{z}^{(0)}\right\rangle_{\omega_{e}=\omega_{g}} \cos \phi \\
& =\frac{\left(A_{\oplus}-\frac{\sqrt{2}}{2} A_{l}\right) \hat{E}_{x}^{(0)} \psi_{0} \Delta z r}{\mu_{0} \hbar \omega_{e}}\left(\frac{\mathscr{A}}{4} \sin \mathscr{P}\right.
\end{aligned}
$$




$$
\begin{aligned}
& \left.+\frac{\mathscr{B}}{2} \cos \mathscr{P}\right) \sin 2 \phi, \\
& n_{\phi-x}^{(1)}=\frac{-1}{2 \mu_{0} \hbar \omega_{e}} \operatorname{Re}\left\langle\tilde{E}_{y}^{(1) *} \tilde{B}_{z}^{(0)}\right\rangle_{\omega_{e}=\omega_{g}} \sin \phi \\
& +\frac{1}{2 \mu_{0} \hbar \omega_{e}} \operatorname{Re}\left\langle\tilde{E}_{z}^{(1) *} \tilde{B}_{x}^{(0)}\right\rangle_{\omega_{e}=\omega_{g}} \cos \phi \\
& +\frac{1}{2 \mu_{0} \hbar \omega_{e}} \operatorname{Re}\left\langle\tilde{E}_{z}^{(1) *} \tilde{B}_{y}^{(0)}\right\rangle_{\omega_{e}=\omega_{g}} \sin \phi \\
& =\frac{A_{x} \hat{E}_{x}^{(0)} \psi_{0} \Delta z r}{\mu_{0} \hbar \omega_{e}}\left\{\frac{\mathscr{A}}{2} \sin \mathscr{P}+\mathscr{B} \cos \mathscr{P}\right\} \sin ^{2} \phi \\
& +\frac{1}{\mu_{0} \hbar \omega_{e}}\left\{A _ { x } \hat { E } _ { x } ^ { ( 0 ) } \operatorname { R e } \left(\frac{1}{\omega_{e}} \exp \left[i\left(k_{g} z-\omega_{g} t\right)\right]^{*}\right.\right. \\
& \left.\left.\cdot F_{2}\right\rangle_{\omega_{e}=\omega_{g}}\right\} \sin \phi \cos ^{2} \phi \\
& +\frac{1}{\mu_{0} \hbar \omega_{e}}\left\{\frac { A _ { x } \hat { E } _ { x } ^ { ( 0 ) } } { 2 } \operatorname { R e } \left(\frac{1}{\omega_{e}} \exp \left[i\left(k_{g} z-\omega_{g} t\right)\right]^{*}\right.\right. \\
& \left.\left.\cdot\left(F_{4}-2 i F_{1}\right)\right|_{\omega_{e}=\omega_{g}}\right\} \sin \phi \\
& -\frac{i}{\mu_{0} \hbar \omega_{e}}\left\{\frac { A _ { x } \hat { E } _ { x } ^ { ( 0 ) } } { 2 } \operatorname { R e } \left(\frac{1}{\omega_{e}} \exp \left[i\left(k_{g} z-\omega_{g} t\right)\right]^{*}\right.\right. \\
& \left.\left.\cdot F_{3}\right\rangle_{\omega_{e}=\omega_{g}}\right\} \sin \phi \cos ^{2} \phi
\end{aligned}
$$

Eqs. (56) and (57) show that the transverse PPFs $n_{\phi-\oplus, l}^{(1)}$ are generated by the combination state of the $\oplus$-type and the $l$ type polarizations, and $n_{\phi-x}^{(1)}$ is produced by the pure $x$-type polarization state.

5. The EM response to the HFGWs in the coupling system between the transverse background static electric field $\hat{E}_{y}^{(0)}$ and the GB.

Then $\hat{E}_{x}^{(0)}=\hat{E}_{z}^{(0)}=\hat{B}_{x}^{(0)}=\hat{B}_{y}^{(0)}=\hat{B}_{z}^{(0)}=0$ and only $\hat{E}_{y}^{(0)} \neq 0$. In this case from Eqs. (20), (45) and (48), in the same way, under the resonance condition $\left(\omega_{e}=\omega_{g}\right)$, the transverse PPFs can be given by

$$
\begin{aligned}
n_{\phi-\otimes}^{(1)}= & \text { replace } \hat{B}_{x}^{(0)} \text { of Eq. (53) by } \hat{E}_{y}^{(0)}, \\
n_{\phi-\oplus, l}^{(1)}= & \frac{-1}{2 \mu_{0} \hbar \omega_{e}} \operatorname{Re}\left\langle\tilde{E}_{y}^{(1) *} \tilde{B}_{z}^{(0)}\right\rangle_{\omega_{e}=\omega_{g}} \sin \phi \\
= & \frac{\left(A_{\oplus}+\frac{\sqrt{2}}{2} A_{l}\right) \hat{E}_{y}^{(0)} \psi_{0} \Delta z r}{\mu_{0} \hbar \omega_{e}}\left(\frac{\mathscr{A}}{2} \sin \mathscr{P}\right. \\
& +\mathscr{B} \cos \mathscr{P}) \sin ^{2} \phi,
\end{aligned}
$$

$$
\begin{aligned}
n_{\phi-y}^{(1)}= & \frac{1}{2 \mu_{0} \hbar \omega_{e}} \operatorname{Re}\left\langle\tilde{E}_{z}^{(1) *} \tilde{B}_{x}^{(0)}\right\rangle_{\omega_{e}=\omega_{g}} \cos \phi \\
& +\frac{1}{2 \mu_{0} \hbar \omega_{e}} \operatorname{Re}\left\langle\tilde{E}_{z}^{(1) *} \tilde{B}_{y}^{(0)}\right\rangle_{\omega_{e}=\omega_{g}} \sin \phi \\
= & \frac{1}{\mu_{0} \hbar \omega_{e}}\left\{A _ { y } \hat { E } _ { y } ^ { ( 0 ) } \operatorname { R e } \left\langle\frac{1}{\omega_{e}} \exp \left[i\left(k_{g} z-\omega_{g} t\right)\right]^{*}\right.\right.
\end{aligned}
$$

$$
\begin{aligned}
& \left.\left.\cdot F_{2}\right\rangle_{\omega_{e}=\omega_{g}}\right\} \sin \phi \cos ^{2} \phi \\
& +\frac{1}{\mu_{0} \hbar \omega_{e}}\left\{\frac { A _ { y } \hat { E } _ { y } ^ { ( 0 ) } } { 2 } \operatorname { R e } \left\langle\frac{1}{\omega_{e}} \exp \left[i\left(k_{g} z-\omega_{g} t\right)\right]^{*}\right.\right. \\
& \left.\left.\cdot\left(F_{4}-2 i F_{1}\right)\right\rangle_{\omega_{e}=\omega_{g}}\right\} \sin \phi \\
& -\frac{i}{\mu_{0} \hbar \omega_{e}}\left\{\frac { A _ { y } \hat { E } _ { y } ^ { ( 0 ) } } { 2 } \operatorname { R e } \left\langle\frac{1}{\omega_{e}} \exp \left[i\left(k_{g} z-\omega_{g} t\right)\right]^{*}\right.\right. \\
& \left.\left.\cdot F_{3}\right\rangle_{\omega_{e}=\omega_{g}}\right\} \sin \phi \cos ^{2} \phi ;
\end{aligned}
$$

Eq. (58) shows that the transverse PPF $n_{\phi-\otimes}^{(1)}$ is generated by the pure $\otimes$-type polarization state; Eq. (59) shows that the transverse PPF $n_{\phi-\oplus, l}^{(1)}$ is generated by the combination state of the $\oplus$-type and the $l$-type polarizations, and Eq. (60) shows that $n_{\phi-y}^{(1)}$ is produced by the pure $y$-type polarization state.

6. The EM response to the HFGWs in the coupling system between the longitudinal background static electric field $\hat{E}_{z}^{(0)}$ and the GB.

Then $\hat{E}_{x}^{(0)}=\hat{E}_{y}^{(0)}=\hat{B}_{x}^{(0)}=\hat{B}_{y}^{(0)}=\hat{B}_{z}^{(0)}=0$ and only $\hat{E}_{z}^{(0)} \neq 0$. In the same way, under the resonance condition $\left(\omega_{e}=\omega_{g}\right)$, the transverse PPFs, can be given by

$$
\begin{aligned}
n_{\phi-x}^{(1)} & =\frac{-1}{2 \mu_{0} \hbar \omega_{e}} \operatorname{Re}\left\langle\tilde{E}_{x}^{(1) *} \tilde{B}_{z}^{(0)}\right\rangle_{\omega_{e}=\omega_{g}} \cos \phi \\
& =\frac{A_{x} \hat{E}_{z}^{(0)} \psi_{0} \Delta z r}{\mu_{0} \hbar \omega_{e}}\left\{\frac{\mathscr{A}}{2} \sin \mathscr{P}+\mathscr{B} \cos \mathscr{P}\right\} \sin 2 \phi, \\
n_{\phi-y}^{(1)} & =\frac{-1}{2 \mu_{0} \hbar \omega_{e}} \operatorname{Re}\left\langle\tilde{E}_{y}^{(1) *} \tilde{B}_{z}^{(0)}\right\rangle_{\omega_{e}=\omega_{g}} \sin \phi \\
& =\frac{A_{y} \hat{E}_{z}^{(0)} \psi_{0} k_{g} \Delta z r}{\mu_{0} \hbar \omega_{e}}\left\{\frac{\mathscr{A}}{2} \sin \mathscr{P}+\mathscr{B} \cos \mathscr{P}\right\} \sin ^{2} \phi,
\end{aligned}
$$

$$
\begin{aligned}
n_{\phi-b, l}^{(1)}= & \frac{1}{2 \mu_{0} \hbar \omega_{e}} \operatorname{Re}\left\langle\tilde{E}_{z}^{(1) *} \tilde{B}_{x}^{(0)}\right\rangle_{\omega_{e}=\omega_{g}} \cos \phi \\
& +\frac{1}{2 \mu_{0} \hbar \omega_{e}} \operatorname{Re}\left\langle\tilde{E}_{z}^{(1) *} \tilde{B}_{y}^{(0)}\right\rangle_{\omega_{e}=\omega_{g}} \sin \phi \\
= & \frac{1}{\mu_{0} \hbar \omega_{e}}\left\{\left(\frac{\sqrt{2}}{2} A_{l}-A_{b}\right) \hat{E}_{z}^{(0)}\right. \\
& \left.\operatorname{Re}\left\langle\frac{1}{\omega_{e}} \exp \left[i\left(k_{g} z-\omega_{g} t\right)\right]^{*} \cdot F_{2}\right\rangle_{\omega_{e}=\omega_{g}}\right\} \sin \phi \cos ^{2} \phi \\
& +\frac{1}{\mu_{0} \hbar \omega_{e}}\left\{\left(\frac{\sqrt{2}}{2} A_{l}-A_{b}\right) \hat{E}_{z}^{(0)}\right. \\
& \left.\operatorname{Re}\left\langle\frac{1}{\omega_{e}} \exp \left[i\left(k_{g} z-\omega_{g} t\right)\right]^{*} \cdot\left(F_{4}-2 i F_{1}\right)\right\rangle_{\omega_{e}=\omega_{g}}\right\} \sin \phi \\
& -\frac{i}{\mu_{0} \hbar \omega_{e}}\left\{\left(\frac{\sqrt{2}}{2} A_{l}-A_{b}\right) \hat{E}_{z}^{(0)}\right. \\
& \left.\operatorname{Re}\left\langle\frac{1}{\omega_{e}} \exp \left[i\left(k_{g} z-\omega_{g} t\right)\right]^{*} \cdot F_{3}\right\rangle_{\omega_{e}=\omega_{g}}\right\} \sin \phi \cos ^{2} \phi
\end{aligned}
$$

Eqs. (61) and (62) show that the transverse PPFs, $n_{\phi-x}^{(1)}$ and $n_{\phi-y}^{(1)}$ are generated by the pure $x$-type polarization and the 
pure $y$-type polarization of the HFGWs, respectively. Equation (63) shows that the PPF $n_{\phi-b, l}^{(1)}$ is produced by the combination state of the $b$-type and the $l$-type polarizations.

In all of the above discussions, the ratio [of the electric component $\left(\tilde{E}_{x}^{(1)}, \tilde{E}_{y}^{(1)}, \tilde{E}_{z}^{(1)}\right)$ to the related magnetic components $\left.\left(\tilde{B}_{x}^{(0)}, \tilde{B}_{y}^{(0)}, \tilde{B}_{z}^{(0)}\right)\right]$ of the PPFs is much less than the ratio of the background noise photon flux. This means that the PPFs expressed by Eqs. (50) to (53), (54) to (60), (61) to (63) have very low wave impedance $[43,59]$ which is much less than the wave impedance to the BPFs (see below). Then the PPFs (i.e., the signal photon fluxes) would be easier to pass through the transmission way of the 3DSR system than the BPFs due to very small Ohm losses of the PPFs.

According to the same way, and from Eqs. (19) and (44), the PPF in the EM response to the HFGWs in the coupling system between the transverse background static magnetic field $\hat{B}_{x}^{(0)}$ and the GB can be given by

$$
\begin{aligned}
n_{\phi-\oplus, b, l}^{(1)}= & \frac{1}{2 \mu_{0} \hbar \omega_{e}} \operatorname{Re}\left\langle\tilde{E}_{z}^{(0) *} \tilde{B}_{x}^{(1)}\right\rangle_{\omega_{e}=\omega_{g}} \cos \phi \\
= & \frac{\left(A_{b}-A_{\oplus}+\sqrt{2} A_{l}\right) \hat{B}_{x}^{(0)} k_{g} \Delta z r}{2 \mu_{0} \hbar \omega_{e}} \\
& \cdot \operatorname{Re}\left\langle F_{1}^{*} \cdot \exp \left[i\left(k_{g} z-\omega_{g} t\right)\right]\right\rangle_{\omega_{e}=\omega_{g}} \cos ^{2} \phi .
\end{aligned}
$$

Equation (64) shows that the PPF $n_{\phi-\oplus, b, l}^{(1)}$, is generated by the combination state among the $\oplus$-type, the $b$-type and the $l$-type polarizations of the HFGWs. Obviously the ratio of the electric component to the magnetic component of the PPF is larger than that of the PPF $n_{\phi-\oplus, b, l}^{(1)}$ [Eq. (52)], i.e., the PPF expressed by Eq. (64) has a larger wave impedance. However, its angular distribution factor $\cos ^{2} \phi$ (see Fig. 6d) is quite different from the $\sin 2 \phi$ of the BPF, Eq. (46), Fig. 7. Thus, it is always possible to distinguish them.

7. Angular distributions of the strengths and the "rotation directions" of typical PPFs in the cylindrical polar coordinates.

Based on the above discussion, the angular distributions of the strengths and the "rotation directions" for some of typical transverse PPFs are listed in the following figures (Fig. 6a-e).

In Fig. $6 \mathrm{a}, n_{\phi}^{(1)} \propto \sin ^{2} \phi$, and it includes the following five cases:

(i) $n_{\phi-\otimes}^{(1)}$, Eq. (50). This is the transverse PPF displaying the pure $\otimes$-type polarization state (the tensor-mode gravitons) of the HFGWs. The PPF is from the EM response to HFGWs in the coupling between transverse static magnetic field $\hat{B}_{y}^{(0)}$ and GB in the 3DSR.

(ii) $n_{\phi-\oplus, b, l}^{(1)}$, Eq. (52). This is the transverse PPF displaying the combination state of the $\oplus$-type, the $b$-type and the $l$ type polarizations (the tensor-mode and the scalar-mode gravitons) of the HFGWs. The PPF is from EM response to HFGWs in coupling between transverse static magnetic field $\hat{B}_{x}^{(0)}$ and GB in the 3DSR.

(iii) $n_{\phi-x}^{(1)}$, Eqs. (54) and (57a). This is the transverse PPF displaying the pure $x$-type polarization state (the vector mode gravitons) of the HFGWs. The PPF is from the EM response to HFGWs in coupling between the longitudinal static magnetic field $\hat{B}_{z}^{(0)}$ (or the transverse static electric field $\hat{E}_{x}^{(0)}$ ) and the GB in the 3DSR.

(iv) $n_{\phi-\oplus, l}^{(1)}$, Eq. (59). This is the transverse PPF displaying the combination state of the $\oplus$-type and the $l$-type polarizations (the tensor-mode and the scalar-mode gravitons) of the HFGWs. The PPF is from the EM response to HFGWs in the coupling between the transverse static electric field $\hat{E}_{y}^{(0)}$ and the GB in the 3DSR.

(v) $n_{\phi-y}^{(1)}$, Eq. (62). This is the transverse PPF displaying the pure $y$-type polarization state (the vector mode gravitons) of the HFGWs. The PPF is from the EM response to HFGWs in the coupling between the longitudinal static electric field $\hat{E}_{z}^{(0)}$ and the GB in the 3DSR.

The best detection position of all of such PPFs should be the receiving surfaces at $\phi=\pi / 2$ and $\phi=3 \pi / 2$ (see Fig. 6a), where the PPFs have their maximum, while the BPF (the background noise photon flux) vanishes at the surface (see Fig. 7). Because the BPF from the GB will be the dominant source of the noise photon fluxes, i.e., other noise photon fluxes [e.g., shot noise, Johnson noise, quantization noise, thermal noise (if operation temperature $T<1 K$ ), preamplifier noise, etc.] are all much less than the BPF [44], in order to detect the PPFs generated by the HFGWs $\left(v \sim 10^{9}\right.$ to $10^{12} \mathrm{~Hz}, h \sim 10^{-21}$ to $10^{-23}$ ) in the braneworld [21], the requisite minimal accumulation time of the signals can be less or much less than $10^{4} s$ [see Appendix B]. Moreover, since the PPFs, Eqs. (50), (52), (54), (59), (62) and the BPF, Eq. (46) also have other very different physical behaviors, such as the wave impedance, decay rate [the decay factor of the PPFs is $\exp \left(-\frac{r^{2}}{W^{2}}\right)$, see Eqs. (50), (52), (59) and (62), while the decay factor of the BPF is $\exp \left(-\frac{2 r^{2}}{W^{2}}\right)$, see Eq. (46)], etc., then the displaying condition to the PPFs can be further relaxed. Besides, the "rotation direction" expressed by Fig. 6a is completely "left-handed circular" or completely "righthanded circular", and the "left-handed circular" or "righthanded circular" property depends on the phase factors in Eqs. (50), (52), (54), (59) and (62).

In Fig. $6 \mathrm{~b}, n_{\phi}^{(1)} \propto \sin 2 \phi$, and it includes the following five cases:

(i) $n_{\phi-\oplus, l}^{(1)}$, Eq. (51), transverse PPF displaying combined $\oplus$ - and $l$-polarizations, by coupling between transverse static magnetic field $\hat{B}_{y}^{(0)}$ and the GB in the 3DSR.

(ii) $n_{\phi-\otimes}^{(1)}$, Eqs. (53) and (58), transverse PPFs displaying pure $\otimes$-polarization, by coupling between transverse 


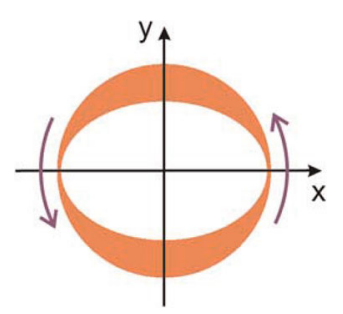

(a)

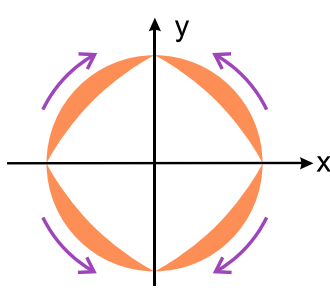

(b)

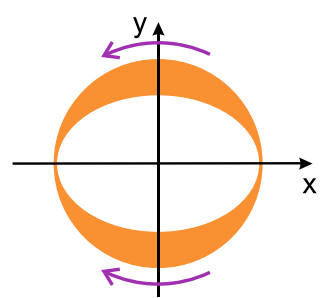

(c)

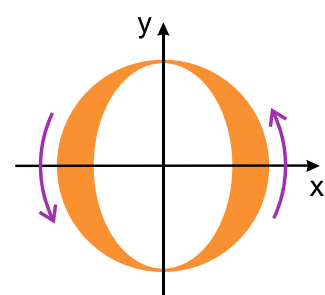

(d)

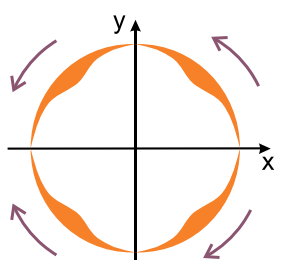

(e)

Fig. 6 The angular distribution (in the cylindrical polar coordinates) of the strengths and "rotational directions" of the PPFs, produced by the HFGWs having different polarization states. Here, for subfigures a-e, we have $n_{\phi}^{(1)} \propto \sin ^{2} \phi, n_{\phi}^{(1)} \propto \sin 2 \phi, n_{\phi}^{(1)} \propto \sin \phi, n_{\phi}^{(1)} \propto \cos ^{2} \phi$, $n_{\phi}^{(1)} \propto \sin \phi \cos ^{2} \phi$, respectively

static magnetic field $\hat{B}_{x}^{(0)}$ (or transverse electric field $\left.\hat{E}_{y}^{(0)}\right)$ and the GB.

(iii) $n_{\phi-y}^{(1)}$, Eq. (55), transverse PPF displaying pure $y$ polarization, by coupling between longitudinal static magnetic field $\hat{B}_{z}^{(0)}$ and the GB.

(iv) $n_{\phi-\oplus, l}^{(1)}$, Eq. (56), transverse PPF displaying combined $\oplus$ - and $l$-polarizations, by coupling between transverse static electric field $\hat{E}_{x}^{(0)}$ and the GB.

(v) $n_{\phi-x}^{(1)}$, Eq. (61), transverse PPF displaying pure $x$ polarization, by coupling between longitudinal static electric field $\hat{E}_{y}^{(0)}$ and the GB.

Unlike Fig. 6a, here the "rotation direction" of the PPFs are not completely "left-handed circular" or not completely "right-handed circular", and it and the transverse BPF have the same angular distribution [see Eq. (46)] and Fig. 7. Thus the displaying condition in Fig. $6 \mathrm{~b}$ will be worse than that in Fig. 6a. However, because the transverse PPFs in Fig. 6b and the BPF have other different physical behaviors, such as the different wave impedance, decay rate, and even different propagating directions in the local region, it is possible to display and distinguish the PPFs from the BPF.

In Fig. $6 \mathrm{c}, n_{\phi}^{(1)} \propto \sin \phi$, and it includes the following three cases:

(i) $n_{\phi-x}^{(1)}$, Eq. $(57 \mathrm{c})$, transverse PPF displaying pure $x$ polarization, by coupling between transverse static electric field $\hat{E}_{x}^{(0)}$ and the GB in the 3DSR.

(ii) $n_{\phi-y}^{(1)}$, Eq. (60b), transverse PPF displaying pure $y$ polarization, by coupling between transverse static electric field $\hat{E}_{y}^{(0)}$ and the GB.

(iii) $n_{\phi-b, l}^{(1)}$, Eq. (63b), transverse PPF displaying combined $b$ - and $l$-polarizations, by coupling between the longitudinal static electric field $\hat{E}_{z}^{(0)}$ and the GB.

Unlike Fig. 6a, here the "rotation direction" of the PPFs is not completely "left-handed circular" or not completely "right-handed circular". However, the best detection position

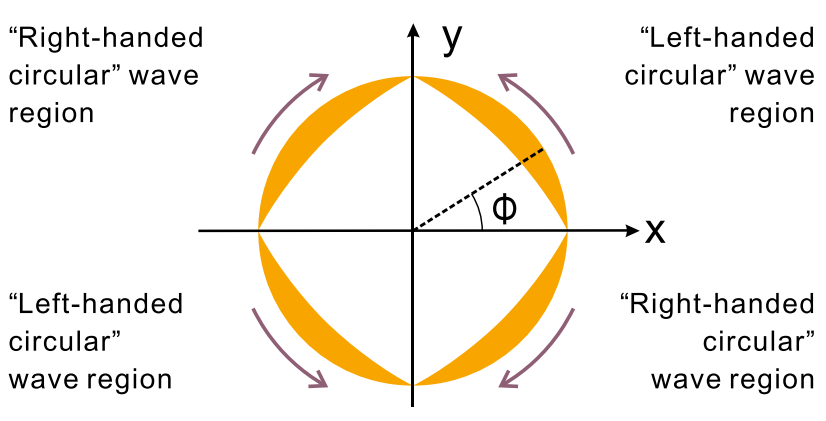

Fig. 7 The strength distribution of transverse background photon flux $n_{\phi}^{(0)}$, Eq. (46), in the cylindrical polar coordinates

of the PPFs is also the receiving surfaces at $\phi=\pi / 2$ and $3 \pi / 2$ [see Fig. 6a, c], where the PPFs have their peak values while the BPF vanishes.

In Fig. 6d, the $n_{\phi-\oplus, b, l}^{(1)} \propto \cos ^{2} \phi$, Eq. (64), and it is the transverse PPF displaying the combination state of the $\oplus$-type (the tensor-mode gravitons) and the $b$-type, $l$-type polarizations (the scalar-mode gravitons) of the HFGWs. The PPF is from the EM response to the HFGWs in the coupling between the transverse static magnetic field $\hat{B}_{x}^{(0)}$ and the GB. It needs to be emphasized that the $n_{\phi-\oplus, b, l}^{(1)}$, Eq. (52), is also the transverse PPF displaying the combination state of the $\oplus$-type, the $b$-type and $l$-type polarizations, but they have different angular distributions. The position of the peak values of $n_{\phi-\oplus, b, l}^{(1)}$, Eq. (52), are surfaces at $\phi=\pi / 2$ and $3 \pi / 2$, while the positions of peak values of $n_{\phi-\oplus, b, l}^{(1)}$, Eq. (64), are surfaces at $\phi=0, \pi$. Especially, the peak value areas of the PPF (signal photon fluxes) are just the zero value area of the BPF (background noise photon flux), Eq. (46) and Fig. 7.

The PPFs, $n_{\phi-\oplus, b, l}^{(1)}$, Eq. (52) and Eq. (64), are from the same EM response to the HFGWs in the coupling between the transverse static magnetic field $\hat{B}_{x}^{(0)}$ and the GB. This means that displaying the PPFs at such areas would show very strong complementarity. Moreover, like the PPFs expressed in Fig. 6a, here, the PPF $n_{\phi-\oplus, b, l}^{(1)}$ is also completely "left- 
handed circular" or completely "right-handed circular" (see Fig. 6d).

In Fig. 6e, $n_{\phi-y}^{(1)} \propto \sin \phi \cos ^{2} \phi$, Eqs. (57b), (57d), (60a) and $(60 \mathrm{c})$, and it is the transverse PPF displaying the pure $x$ type polarization (vector-mode) of the HFGWs and the pure $y$-type polarizations (vector-mode) of the HFGWs, respectively. For the former, the PPF is from the EM response to the HFGWs in the coupling between the transverse static electric field $\hat{E}_{x}^{(0)}$ and the GB. For the latter, the PPF is from the EM response to the HFGWs in the coupling between the transverse static electric field $\hat{E}_{y}^{(0)}$ and the GB. Clearly, the term $n_{\phi-x}^{(1)}$ expressed by Eqs. (57b), (57d) and $n_{\phi-y}^{(1)}$ expressed by Eqs. (60a), (60c) are not completely "left-handed circular" or completely "right-handed circular". Besides, its angular distribution factor $\sin \phi \cos ^{2} \phi=\frac{1}{2} \sin 2 \phi \cos \phi$ is smaller than $\sin 2 \phi$, Eq. (46) of the BPF. Thus, distinguishability of $n_{\phi-x}^{(1)}$ and $n_{\phi-y}^{(1)}$ in Fig. 6e is worse than the PPFs in Figs. 6ad. Nevertheless, considering the obvious difference of other physical behaviors (e.g., wave impedance, decay rate, and propagating direction) between the PPFs in Fig. 6e and the BPF in Fig. 7, to distinguish them is still possible.

The above discussions show that the three polarization states (the $\otimes$-type, the $x$-type and the $y$-type polarizations, i.e., the tensor-mode and the vector-mode gravitons) of the HFGWs can be clearly separated and distinguished. On the other hand, $\oplus$-type polarization (the tensor-mode gravitons), the $b$-type and the $l$-type polarizations (the scalar-mode gravitons) of the HFGWs are often expressed as their combination states to generate the PPFs. However, from the PPFs produced by these combination states, it is easy to calculate the PPFs generated by the pure $\oplus$-type, the pure $b$-type and the pure $l$-type polarizations, and thus we can completely determine these polarizations, respectively.

From Eqs. (51) to (52) and (63b), we have

$$
\begin{aligned}
n_{\phi-\oplus, b, l}^{(1)} & =-n_{\phi-\oplus}^{(1)}+n_{\phi-b}^{(1)}+\sqrt{2} n_{\phi-l}^{(1)}, \\
n_{\phi-\oplus, l}^{(1)} & =n_{\phi-\oplus}^{(1)}+\frac{\sqrt{2}}{2} n_{\phi-l}^{(1)}, \\
n_{\phi-b, l}^{(1)} & =-n_{\phi-b}^{(1)}+\frac{\sqrt{2}}{2} n_{\phi-l}^{(1)},
\end{aligned}
$$

where $n_{\phi-\oplus, b, l}^{(1)}$, Eq. (52), $n_{\phi-\oplus, l}^{(1)}$, Eq. (51), and $n_{\phi-b, l}^{(1)}$, Eq. (63b) are the PPFs generated by the combination state of the $\oplus$-type, the $b$-type, the $l$-type polarizations, by the combination state of the $\oplus$-type, the $l$-type polarizations, and by the combination state of the $b$-type, the $l$-type polarizations, respectively.

Clearly, $n_{\phi-\oplus}^{(1)}, n_{\phi-b}^{(1)}$ and $n_{\phi-l}^{(1)}$ in Eq. (65) are the PPFs generated by the pure $\oplus$-type, the pure $b$-type and the pure $l$-type polarizations of the HFGWs, respectively. By using Eq. (65), it is easy to find

$n_{\phi-\oplus}^{(1)}=-\frac{1}{2}\left(n_{\phi-\oplus, b, l}^{(1)}-n_{\phi-\oplus, l}^{(1)}+n_{\phi-b, l}^{(1)}\right)$,

$$
\begin{aligned}
& n_{\phi-b}^{(1)}=\frac{1}{2}\left(n_{\phi-\oplus, b, l}^{(1)}-n_{\phi-\oplus, l}^{(1)}-3 n_{\phi-b, l}^{(1)}\right), \\
& n_{\phi-l}^{(1)}=\frac{1}{\sqrt{2}}\left(n_{\phi-\oplus, b, l}^{(1)}-n_{\phi-\oplus, l}^{(1)}+n_{\phi-b, l}^{(1)}\right) .
\end{aligned}
$$

Notice that the each term $n_{\phi-\oplus, b, l}^{(1)}, n_{\phi-\oplus, l}^{(1)}$ and $n_{\phi-b, l}^{(1)}$ of the right side in Eqs. (66), (67) and (68) are directly measurable physical quantities. Therefore, the values and propagating directions of $n_{\phi-\oplus}^{(1)}, n_{\phi-b}^{(1)}$ and $n_{\phi-l}^{(1)}$ can be completely confirmed.

So far the PPFs produced by the six polarization states (the $\otimes$-type, the $x$-type, the $y$-type, the $\oplus$-type, the $b$-type and the $l$-type polarizations) of the HFGWs can be calculated and completely confirmed [see, e.g., Eqs. (50), (54), (62), (66), (67) and (68), respectively]. In other words, in principle, the six polarizations of the HFGWs can be clearly displayed and distinguished in the EM response of our 3DSR system.

\section{Numerical estimations of the perturbative photon fluxes in the 3DSR system}

Unlike the EM response to the HFGWs in the galacticextragalactic background EM fields, the 3DSR is a closed cryogenic system with vacuum, which is shielded and isolated by superconductor materials from outside world. The EM response to the HFGWs in the 3DSR system has the following important characteristics:

(i) Since shielding of the 3DSR system to the EM fields from outside region, the large space-accumulation effect of the PPFs cannot enter and influence the EM fields inside the 3DSR system. However, the superconductor and the shell of the 3DSR system are transparent to the HFGWs.

(ii) Unlike the galactic-extragalactic background magnetic fields $\left(\hat{B}_{x}^{(0)} \sim 10^{-9}\right.$ to $\left.10^{-11} T\right)$, the background static magnetic fields $\hat{B}^{(0)}$ of the 3DSR system can reach up to $\sim 10 T$ or larger. The cooperation institute (High Magnetic Field Laboratory, Chinese Academy of Sciences) of our research team has been fully equipped with ability to construct the superconducting magnet [60], and it is also the builder of the superconducting magnet for the Experimental Advanced Superconducting Tokamak (EAST) for controlled nuclear fusion. The magnet can generate a static magnetic field with $\hat{B}^{(0)}=10 T$ in an effective cross section with diameter of at least 80 to $100 \mathrm{~cm}$, and operation temperature can be reduced to $1 K$ or less. Obviously, such a magnetic field is much stronger than the galactic-extragalactic background magnetic fields, although the typical spatial dimension of the former is only of the order of magnitude of a meter (typical laboratory dimension). 
(iii) The major noise sources of the EM signals from the case of galactic-extragalactic EM fields (see below) would be from the space microwave background, and the key noises of the case of the laboratory-based high magnet are from the microwave photons inside the 3DSR system, which are almost independent of the space background EM noise and the cosmic dusts.

(iv) Because the PPFs are first-order perturbations to the background EM fields and not second-order perturbations, the PPFs, Eqs. (50) to (53), (54) to (60) and (61) to (64), in the 3DSR system are proportional to the amplitudes themselves $(h)$ of the HFGWs and not their square $h^{2}$; see, e.g., Eqs. (34) and (37) (i.e., they could be the PPFs in the galactic-extragalactic background magnetic fields). Then the parameter $h \hat{B}^{(0)}$ in the first-order PPFs [see, e.g., Eqs. (50) and (51)] will be much larger than the parameter $\left(h \hat{B}^{(0)}\right)^{2}$ in the second-order PPFs [see, e.g., Eqs. (24) and (25)]. This property effectively compensates for the weakness of the far-field amplitudes of the HFGWs in the 3DSR system. Of course, the PPFs in the 3DSR system are always accompanied by the noise photons inside the system, which mainly are from the background photon fluxes caused by the GB. Thus, in order to identify the total signal photon flux at an effective receiving surface $\Delta s$, the time accumulation effect of the PPF must be larger than the effect of the noise photon flux fluctuation at the receiving surface $\Delta s$.

As mentioned above, in order to display the relatively weak PPFs in the background noise photon fluxes (BPFs), we need a long enough accumulation time of the signal [see Appendix B]. For the typical parameters of the HFGWs predicted by the braneworld scenarios [9,21], the observing and distinguishing of the HFGWs would be quite well possible due to their large amplitudes, higher frequencies and the discrete spectrum characteristics. The measurement of the relic HFGWs will face a big challenge too, but it is not impossible (see Table 2).

Table 2 shows the displaying conditions of the HFGWs for some typical cosmological models and high-energy astrophysical processes, where $n_{\phi \text { (total) }}^{(1)}$ is the total signal photon flux at the receiving surface $\Delta s\left(\Delta s \sim 3 \times 10^{-2} \mathrm{~m}^{2}\right)$, and $n_{\text {total }}^{(0)}$ is the allowable upper limit of the total noise photon flux at the surface $\Delta s$ for various values of the HFGW amplitudes, and $\Delta t_{\min }$ [see Eq. (B.8) in Appendix B] is the requisite minimal accumulation time of the signals; $v_{e}=v_{g}=3 \times 10^{9}$ $\mathrm{Hz}$ or $3 \times 10^{8} \mathrm{~Hz}$ (the resonance frequency); the background static magnetic field $B^{(0)}$ is $10 \mathrm{~T}$; the interaction dimension $\Delta z$ is $60 \mathrm{~cm}$ (typical dimension of the static magnetic field in our 3DSR); the power of the Gaussian beam is $\sim 10 \mathrm{~W}$ and the operation temperature should be less than $1 \mathrm{~K}$.
Notice that, for the GB of $P \sim 10 W$ in the 3DSR, the maximum of $n_{\phi(\text { total })}^{(0)}$ at the receiving surface $\Delta s=3 \times$ $10^{-2} \mathrm{~m}^{2}$ is about $\sim 10^{22} \mathrm{~s}^{-1}$. This means that even if the peak values of the noise photon flux and the PPFs appear at the same receiving surface (see, e.g., Fig. 6b), the $\Delta t_{\min }$ can be limited in $\sim 10^{4} s$ (e.g. the first case in Table 2). In fact, in many cases discussed in this paper, the peak position of two such kinds of photon fluxes do not appear at the same receiving surface, and especially, the peak value positions (see, e.g., Fig. 6a,c, d) of the signal photon fluxes are just the zero value areas $(\phi=0, \pi / 2, \pi, 3 \pi / 2)$ of the background noises photon flux $n_{\phi}^{(0)}$ (see Fig. 7). In this case the displaying condition can be further relaxed.

In Table $2, v \sim 3 \times 10^{8}$ to $3 \times 10^{9} \mathrm{~Hz}$ is a typical peak value region of the spectrum of the HFGWs in the braneworld scenarios [21], the short-term anisotropic inflationary model [32], the pre-big-bang model [27,28] and the quintessential inflationary model [29-31].

It is very interesting to compare the displaying and distinguishing conditions for the case in the galactic-extragalactic fields (e.g. see part 2 of this section and Sect. 3) and in the 3DSR (Sect. 4). In Sect. 3, the large space-accumulation effect caused by the galactic-extragalactic fields is discussed, and Sect. 4 shows the longer time accumulation effect in the strong background static magnetic field and the resonance response of the GB in the 3DSR. They all can effectively compensate for the weakness of the HFGW amplitudes. Clearly, they would be highly complementary to each other. Moreover, the PPFs produced in the 3DSR system by the relic HFGWs in the quintessential inflationary, the pre-big-bang and the short-term anisotropic inflationary models would only be $10^{2} s^{-1}$ to $10^{6} s^{-1}$ (see Table 2), which are much less than the maximum of the transverse BPF (noise photon flux). However, using very different physical behaviors between the PPFs and the BPFs, especially their very different strength distributions and propagating directions [see, e.g., Figs. 6a, b, d,7 and Eqs. (B.10) and (B.11) in Appendix B], it is still possible to reduce the BPF to the allowable upper limits at a suitable receiving surface (see Table 2). Considering their very different wave impedances (four orders of magnitude at least [43]), the displaying of the transverse PPFs produced by the relic HFGWs, in principle, may also be possible though one faces a big challenge here too.

\section{Concluding remarks}

In this paper, for the first time we address the perturbative photon fluxes of the HFGWs having additional polarization states in the EM response, and it shows the separability and detectability of the possible six polarization states of the HFGWs in the EM response. 
Table 2 Displaying condition in 3DSR system (in laboratory scale) for the HFGWs (having possible additional polarizations) from some typical cosmological models and high-energy astrophysical process

\begin{tabular}{|c|c|c|c|c|c|}
\hline $\begin{array}{l}\text { Amplitude(A) } \\
\text { dimensionless }\end{array}$ & Resonance frequency & $n_{\phi(\text { total })}^{(1)}\left(s^{-1}\right)$ & $\Delta t_{\min }(s)$ & $\begin{array}{l}\text { Allowable upper limit } \\
\text { of noise photon flux } \\
n_{\phi \text { (total) }}^{(0)}\left(s^{-1}\right)\end{array}$ & $\begin{array}{l}\text { Possible verifiable cosmo- } \\
\text { logical models and astro- } \\
\text { physical process }\end{array}$ \\
\hline $10^{-23}$ & $3 \times 10^{9} \mathrm{~Hz}$ & $\sim 10^{9}$ & $\sim 10^{4}$ & $\sim 10^{22}$ & Braneworld [21] \\
\hline $10^{-25}$ & $3 \times 10^{9} \mathrm{~Hz}$ & $\sim 10^{7}$ & $\sim 10^{4}$ & $\sim 10^{18}$ & Braneworld [21] \\
\hline $10^{-26}$ & $3 \times 10^{8} \mathrm{~Hz}$ & $\sim 10^{6}$ & $\sim 10^{5}$ & $\sim 10^{18}$ & Short-term anisotropic inflation [32] \\
\hline $10^{-27}$ & $3 \times 10^{9} \mathrm{~Hz}$ & $\sim 10^{5}$ & $\sim 10^{5}$ & $\sim 10^{16}$ & $\begin{array}{l}\text { Interaction of astrophysical plasma } \\
\text { with intense EM radiation [33] }\end{array}$ \\
\hline $10^{-29}$ & $3 \times 10^{9} \mathrm{~Hz}$ & $\sim 10^{3}$ & $\sim 10^{6}$ & $\sim 10^{12}$ & Pre-big-bang $[27,28]$ \\
\hline $10^{-30}$ & $3 \times 10^{9} \mathrm{~Hz}$ & $\sim 10^{2}$ & $\sim 10^{6}$ & $\sim 10^{10}$ & $\begin{array}{l}\text { Quintessential inflationary [29-31] } \\
\text { or upper limit of ordinary inflation- } \\
\text { ary }[25,26]\end{array}$ \\
\hline
\end{tabular}

1. For the EM response to the HFGWs in the 3DSR system and for the EM response to the HFGWs in the galacticextragalactic background stable EM fields, the pure $\otimes$-type polarization (the tensor-mode gravitons), the pure $x$-type and the pure $y$-type polarization (the vector-mode gravitons) of the HFGWs can independently generate the PPFs. The $\oplus$ type polarization (the tensor-mode gravitons), the $b$-type and the $l$-type polarization (the scalar-mode gravitons) in the EM response produce the PPFs in their different combination states. However, the EM perturbations generated by the pure $\oplus$-type, the pure $b$-type and the pure $l$-type polarizations can be directly calculated and can be completely determined from such combination states, respectively. Therefore, all six possible polarization states of the HFGWs may in principle show separability and detectability.

2. The coupling between the background longitudinal static EM fields $\hat{E}_{z}^{(0)}, \hat{B}_{z}^{(0)}$ and the GB in the 3DSR case would only produce the EM response to the $x$-type, the $y$-type polarizations and the combination state of the $b$-type and the $l$-type polarizations [see Eqs. (54), (55), (61), (62) and (63a) to (63c)]. The interaction of the HFGWs with the galacticextragalactic longitudinal stable EM fields $\hat{E}_{z}^{(0)}, \hat{B}_{z}^{(0)}$ would only generate the EM response to the $x$-type and the $y$-type polarizations of the HFGWs [see Eqs. (34) and (35)]. In other words, the longitudinal background static EM fields only produce an EM response to the additional polarizations (the $x$ type, the $y$-type, the $b$-type and the $l$-type polarization states) of the HFGWs, and do not generate any EM response to the traditional $\otimes$-type or $\oplus$-type polarizations in the GR framework. This would provide an effective way to display the pure additional polarization states of the HFGWs.

3. The PPFs [the EM perturbative power fluxes; see Eqs. (38) to (42)] generated by the HFGWs having additional polarization states have the potential to be larger than the PPFs generated by the HFGWs in the framework of the GR [e.g., when $A_{x}, A_{y}, A_{b}$ and $A_{z}$ are equal to zero in
Eqs. (38) to (42)]. This means that the former could cause larger GW radiation damping than the latter for the local GW sources surrounded by stable magnetic fields (e.g. the galactic-extragalactic magnetic fields). If the HFGWs have no additional polarization states, or these additional polarizations are much smaller than the tensorial polarizations, and their effects can be neglected, then the radiation damping would have good self-consistency with the GR. Meanwhile, if the proportions of these nontensorial polarizations are still unknown or with some uncertainty, different cases for the radiation damping may be a potential interesting phenomenon that is worth exploring, and it is possible to find effective constraints to the extra polarization states or to give a possible upper limit of them.

4. In a specific transverse direction, i.e., the $x$-direction in the 3DSR system discussed in this paper, the BPF $n_{x}^{(0)}=0$. However, the PPFs $n_{x}^{(1)}$ propagating along the $x$-direction have significant non-vanishing values [see Eqs. (B.10) to (B.12) in Appendix B]. In this case, the displaying condition in Table 2 can be greatly relaxed. In other words, the PPFs produced by the HFGWs from the braneworld [9,21] by HFGWs predicted by the short-term anisotropic inflationary model [32] and by HFGWs from the interaction [33] of astrophysical plasma with intense EM waves can almost be instantaneously displayed; the requisite minimal accumulation time of the signals for displaying relic HFGWs expected by the pre-big-bang $[27,28]$ and by the quintessential inflationary [29-31] models, would be further relaxed effectively. Thus, utilizing a highly orientational receiving surface to display the PPFs will be very useful.

5. The longitudinal perturbative electric field $\tilde{E}_{z}^{(1)}$ plays an important role in the 3DSR system, i.e., the PPFs [see Eqs. (57b) to (57d) and Eqs. (63a) to (63c)] produced by the resonance effect between the GB and the longitudinal perturbative electrical field $\tilde{E}_{z}^{(1)}$ can effectively display and distinguish the additional polarization states (the $x$-type, the 
$b$-type and the $l$-type polarizations) of the HFGWs. Whereas in the EM response of the galactic-extragalactic background stable electric field $\hat{E}^{(0)}$ to the HFGWs the average values of the transverse PPFs are equal to zero [see Eq. (43)] due to the phase difference of $\pi / 2$ between the longitudinal perturbative electric field, Eq. (20), and the transverse perturbative magnetic fields, Eqs. (17) and (19), the longitudinal perturbative electric field $\tilde{E}_{z}^{(1)}$ does not provide any contribution for the PPFs in such case.

6. In the EM response to the HFGWs in the 3DSR system, the number of PPFs [see, e.g., Eqs. (50), (51)] is independent of the frequency due to $k_{g}=\omega_{g} / c$ [i.e., the related strengths of the signal EM power fluxes are proportional to the frequencies of the HFGWs]. In the EM response to the HFGWs in galactic-extragalactic stable magnetic fields, the strengths of the PPFs [see, e.g., Eqs. (24), (25)] are not only proportional to the square of amplitudes of the HFGWs, but also proportional to their frequencies due to $k_{g}=\omega_{g} / c$ [i.e., the related strengths of the signal EM power fluxes are proportional to the square of their frequencies]. Therefore, very high frequency can effectively compensate the weak HFGW amplitudes. Especially for the HFGWs predicted by the braneworld scenarios [21], since the amplitudes of such HFGWs almost have the same order of magnitude in the frequency band of $\sim 10^{9} \mathrm{~Hz}$ to $10^{12} \mathrm{~Hz}$, and because of $\hbar \omega_{g}=K T$ ( $\mathrm{K}$ is the Boltzmann constant), the power of the PPFs of $10^{12} \mathrm{~Hz}$ will be roughly $10^{3}$ times larger than that of the PPFs of $10^{9} \mathrm{~Hz}$, and the requirement of the operating temperature can be relaxed to $140 \mathrm{~K}$ from $0.14 \mathrm{~K}$. In this case, for the background photon flux $n_{\phi}^{(0)}$ having the same power, the value of $n_{\phi}^{(0)}$ of $10^{12} \mathrm{~Hz}$ will be reduced $10^{3}$ times for an $n_{\phi}^{(0)}$ of $10^{9} \mathrm{~Hz}$. Then the requisite minimal accumulation time of the signals will be reduced by almost one thousand times [see Eq. (B.8)].

Besides, we need to mention two points:

(1) Since the 3DSR is a detection system fixed on the Earth, the 3DSR will have a rotation period of 24 hours to any GW sources in the local space region due to the rotation of the Earth. Thus the background "transverse" and "longitudinal" EM fields in the 3DSR will be periodically changed with the rotation of the Earth. In fact, this is an issue on the relationship between the characteristic parameters (i.e., each polarization component) of the HFGWs and the coordinate rotation. In our 3DSR system, this is an issue on the relationship between the EM response to the HFGWs and the coordinate rotation. Nevertheless, the 3DSR has a special and definite direction, namely, the positive direction of the symmetrical axis of the GB (i.e. the z-direction in Sect. 4). We have showed [41] that only when the propagating direction of the HFGWs and the positive direction of the sym- metrical axis of the GB are the same, the PPFs reach their maximum. If they are perpendicular or opposite to each other, then the PPFs will be one or two orders of magnitude smaller than their peak values. Thus we can determine the propagating direction of the HFGWs by the peak moments of PPFs. The preliminary calculations show that within about two hours around the peak moments, the PPFs can basically maintain the intensity at the same orders of magnitude as the peak values. This means that we can maintain an effective signal accumulation time of $\sim 7 \times 10^{3} s$ for the PPFs, i.e., it basically approaches $10^{4} s$ in Table 2. This is satisfactory. Moreover, the 3DSR is an EM detection system in small scale (order of magnitude of a meter). Therefore, it can rotate not only in the meridional direction (adjusting the polar angle), but also in the latitudinal direction (adjusting the azimuth angle), based on techniques such as laser gyro positioning and some other direction positioning and tracking methods. Thus its spatial orientation can also be effectively adjusted to the best or near optimal direction for the possible HFGW sources.

(2) The detection frequency band of the 3DSR is $\sim 10^{8} \mathrm{~Hz}$ to $10^{12} \mathrm{~Hz}$ or higher, and the observational frequencies (for EM signals) of the FAST is $\sim 7 \times 10^{7}$ to $3 \times 10^{9} \mathrm{~Hz}$ $[55,61]$. This means that the detection frequency bands of the 3DSR and the FAST are overlapping partly in the $\mathrm{GHz}$ band. Therefore, the cooperation and coincidence experiments of them will have very strong complementarity as regards to distinguish and display the possible all six polarizations of the HFGWs in microwave frequency band.

The two points mentioned above will be discussed and studied in detail elsewhere.

Acknowledgements The work of this article is supported by the National Natural Science Foundation of China (Grant No.11605015, No.11375279, No.11873001, No.11947406), the Research Funds for the Central Universities (2019CDXYWL0029), the Foundation of China Academy of Engineering Physics No.2008 T0401 and T0402, the Science and Technology Research Program of Chongqing Municipal Education Commission (Grant No. KJQN201800105), and Natural Science Foundation Project of Chongqing cstc2018jcyjAX0767. We are very grateful for helpful discussions with Prof. Robert M. L. Baker, Prof. Guang-Li Kuang, Prof. Lian-Fu Wei, Prof. C. Corda, Prof. YunFei Tan, Dr. Gary V. Stephenson, Dr. Andrew Beckwith and Dr. Eric W. Davis.

Data Availability Statement This manuscript has no associated data or the data will not be deposited. [Authors' comment: The plan of 3DSR detector is still in progress, and there are no data so far.]

Open Access This article is licensed under a Creative Commons Attribution 4.0 International License, which permits use, sharing, adaptation, distribution and reproduction in any medium or format, as long as you give appropriate credit to the original author(s) and the source, provide a link to the Creative Commons licence, and indicate if changes 
were made. The images or other third party material in this article are included in the article's Creative Commons licence, unless indicated otherwise in a credit line to the material. If material is not included in the article's Creative Commons licence and your intended use is not permitted by statutory regulation or exceeds the permitted use, you will need to obtain permission directly from the copyright holder. To view a copy of this licence, visit http://creativecomm ons.org/licenses/by/4.0/.

Funded by $\mathrm{SCOAP}^{3}$.

Appendix A: a new group of special solutions of the Gaussian-type photon flux for the Helmholtz equation

In this paper, the 3DSR is actually a coupling system between the background static EM fields and the Gaussian-type photon flux (Gaussian beam). Under the condition of the resonance response to the HFGWs, the PPFs (the signal photon fluxes) and the BPFs (including other noise photons) have very different physical behaviors in the local regions. Thus it makes the 3DSR system have a very low standard quantum limit. In Ref. [58] we have given several coupling forms between the GB and the background static magnetic field. Here we select a new group of solutions of the Helmholtz equation, and give their complete expressions. Moreover, in order to display and distinguish effectively all six polarization states of the HFGWs, here such GB does not only couple with the transverse static EM fields, but also the longitudinal static EM fields. In this case, it is possible to display and distinguish all of the different polarization states of the HFGWs.

The general form of the circular-mode GB of the fundamental frequency is [57]

$$
\begin{aligned}
\psi= & \frac{\psi_{0}}{\sqrt{1+(z / f)^{2}}} \exp \left(\frac{-r^{2}}{W^{2}}\right) \exp \left\{i \left[\left(k_{e} z-\omega_{e} t\right)\right.\right. \\
& \left.\left.-\tan ^{-1} \frac{z}{f}+\frac{k_{e} r^{2}}{2 R}+\delta\right]\right\},
\end{aligned}
$$

where $\psi_{0}$ is the amplitude of electrical field of the GB, $f=$ $\pi W_{0}^{2} / \lambda_{e}, W=W_{0} \sqrt{1+(z / f)^{2}}, R=z+f^{2} / z$. The $W_{0}$ is the minimum spot radius, $R$ is the curvature radius of the wave front of the GB at $z, \omega_{e}$ is the angular frequency, $\lambda_{e}$ is the EM wavelength, the $z$-axis is the symmetrical axis of the $\mathrm{GB}$, and $\delta$ is a phase factor.

According to Eq. (A.1) and using the non-divergence condition $\nabla \cdot \tilde{\mathbf{E}}^{(0)}=0$ in the free space, and $\tilde{\mathbf{B}}^{(0)}=$ $-\mathrm{i} / \omega_{\mathrm{e}} \cdot \nabla \times \tilde{\mathbf{E}}^{(0)}$, with the group of special solutions we selected, the components of the GB in the Cartesian coordinates can be given by

$$
\begin{aligned}
\tilde{E}_{x}^{(0)}= & \psi_{e x}=\psi=\frac{\psi_{0}}{\sqrt{1+(z / f)^{2}}} \\
& \exp \left(\frac{-r^{2}}{W^{2}}\right) \exp \left\{i \left[\left(k_{e} z-\omega_{e} t\right)\right.\right.
\end{aligned}
$$

$$
\begin{aligned}
& \left.\left.-\tan ^{-1} \frac{z}{f}+\frac{k_{e} r^{2}}{2 R}+\delta\right]\right\}, \\
& \tilde{E}_{y}^{(0)}=\psi_{e y}=0 \text {, } \\
& \tilde{E}_{z}^{(0)}=\psi_{e z}=2 x F_{1}\left(\mathbf{x}, k_{e}, W\right)=2 r \cos \phi F_{1}\left(\mathbf{x}, k_{e}, W\right) \text {, } \\
& \tilde{B}_{x}^{(0)}=\psi_{b x}=-\frac{i}{\omega_{e}} \frac{\partial \psi_{e z}}{\partial y}=\frac{\sin 2 \phi}{\omega_{e}} F_{2}\left(x, k_{e}, w\right), \\
& \tilde{B}_{y}^{(0)}=\psi_{b y}=-\frac{i}{\omega_{e}}\left(\frac{\partial \psi_{e x}}{\partial z}-\frac{\partial \psi_{e z}}{\partial x}\right) \\
& =\frac{1}{\omega_{e}}\left[F_{4}\left(\mathbf{x}, k_{e}, W\right)\right. \\
& \left.-i\left(2 F_{1}\left(\mathbf{x}, k_{e}, W\right)+\cos ^{2} \phi F_{3}\left(\mathbf{x}, k_{e}, W\right)\right)\right], \\
& \tilde{B}_{z}^{(0)}=\psi_{b z}=\frac{i}{\omega_{e}} \frac{\partial \psi_{e x}}{\partial y} \\
& =\frac{-\sin \phi}{\omega_{e}}\left[\frac{k_{e} r}{R}+\frac{i 2 r}{W_{0}^{2}\left[1+(z / f)^{2}\right]}\right] \psi, \\
& \text { where } F_{1}=\int\left(\frac{1}{W^{2}}-i \frac{k_{e}}{2 R}\right) \psi d z \text {, } \\
& F_{2}=\int\left\{\frac{3 k_{e} r}{W_{0}^{2}\left[1+(z / f)^{2}\right] R}\right. \\
& \left.+i\left[\frac{k_{e}^{2} r}{2 R}+\frac{2 r}{W_{0}^{4}\left[1+(z / f)^{2}\right]}\right]\right\} \psi d z, \\
& F_{3}=\int\left(\frac{k_{e}^{2} r}{2 R}-\frac{2 r^{2}}{W^{4}}+i \frac{6 k_{e} r^{2}}{W^{2} R}\right) \psi d z, \\
& F_{4}=\left\{k_{e}-\frac{1}{f+z^{2} / f}-\frac{k_{e} r^{2}\left[1-(f / z)^{2}\right]}{2 R^{2}}\right. \\
& \left.+i\left[\frac{z}{f^{2}\left[1+(z / f)^{2}\right]}+\frac{2 r^{2}}{W^{2} R}\right]\right\} \psi .
\end{aligned}
$$

According to Eqs. (A.1)-(A.7), we obtained the strength distribution (see Fig. 7) of the transverse background photon flux $n_{\phi}^{(0)}$ in the cylindrical polar coordinates. As demonstrated in this paper, the PPFs (signal photon fluxes) and the $\mathrm{BPF}$ (the dominated noise photon flux) have very different physical behaviors (such as the strength distribution, propagating direction, decay rate, and wave impedance) in the local regions. This is one important physical mechanism of the displayability and separability between the PPFs and the $\mathrm{BPF}$, including the different polarizations of the HFGWs.

\section{Appendix B: Displaying conditon in the background noise photon flux fluctuation.}

Here we briefly discuss the displaying condition in the proposed 3DSR system based on previous works [42,43]. Unlike the B-mode experiment in CMB for the very-low-frequency relic GWs (where the major noise is from the cosmic dust), here the noise sources would be the microwave noise photons inside the 3DSR, and they are almost independent of the cosmic dust. These noise photons include the BPF caused by the GB, and other noise photon fluxes (shot noise, Johnson noise, quantization noise, thermal noise, preamplifier noise, 
diffraction noise, etc.). However, the above-mentioned other noise photon fluxes are all much less than the BPF if the operation temperature $T<1 K$ for the thermal noise [44]. Thus, although the signal photon fluxes are always accompanied by such noise photons, our attention can mainly focus on the BPF fluctuation caused by the GB. In other words, once the PPFs can be displayed in the BPF fluctuation, the influence of all other noise photon flux fluctuations can be neglected.

The displaying condition in the noise photon flux fluctuation is given by

$$
\begin{aligned}
n_{\phi(\text { total })}^{(1)} \Delta t & \geqslant \sqrt{n_{\phi(\text { total })}^{(0)} \Delta t}, \text { then } \Delta t \\
& \geqslant n_{\phi(\text { total })}^{(0)} /\left[n_{\phi(\text { total })}^{(1)}\right]^{2}=\Delta t_{\text {min }},
\end{aligned}
$$

where $\Delta t_{\min }$ is the requisite minimal accumulation time of the signals, and

$n_{\phi(\text { total })}^{(1)}=\int_{\Delta s} n_{\phi}^{(1)} d s$ and $n_{\phi(\text { total })}^{(0)}=\int_{\Delta s} n_{\phi}^{(0)} d s$

are the total signal photon flux and the total noise photon flux passing through the receiving surface $\Delta s$, respectively.

It should be pointed out that, being a very important feature of the BPF expressed by the Gaussian-type wave beam solutions, Eqs. (A.2) to (A.7), there is no BPF $n_{x}^{(0)}$ propagating along the $x$-direction (i.e., a direction perpendicular to the symmetrical axis $z$ of the GB) due to $\tilde{E}_{y}^{(0)}=\psi_{e y}=0$ [see Eq. (A.2)], namely,

$n_{x}^{(0)}=\frac{\operatorname{Re}\left\langle\tilde{E}_{y}^{(0) *} \tilde{B}_{z}^{(0)}\right\rangle}{2 \mu_{0} \hbar \omega_{e}}=\frac{1}{2 \mu_{0} \hbar \omega_{e}} \operatorname{Re}\left\langle\tilde{\psi}_{e y}^{*} \psi_{b z}\right\rangle=0$.

However, the PPFs $n_{x}^{(1)}$ propagating along the $x$-direction has significant non-vanishing values. According to Eqs. (18) and (45), we have

$$
\begin{aligned}
n_{x-\otimes}^{(1)}= & \frac{1}{2 \mu_{0} \hbar \omega_{e}} \operatorname{Re}\left\langle\tilde{E}_{y}^{(1) *} \tilde{B}_{z}^{(0)}\right\rangle_{\omega_{e}=\omega_{g}}=\frac{1}{\mu_{0} \hbar \omega_{e}} \\
& \cdot\left\{\frac{A_{\otimes} \hat{B}_{y}^{(0)} \psi_{0} k_{g} \Delta z r}{2\left[1+(z / f)^{2}\right]^{\frac{1}{2}}\left(z+f^{2} / z\right)} \sin \mathscr{P}\right. \\
& \left.+\frac{A_{\otimes} \hat{B}_{y}^{(0)} \psi_{0} \Delta z r}{W_{0}^{2}\left[1+(z / f)^{2}\right]^{\frac{3}{2}}} \cos \mathscr{P}\right\} \exp \left(-\frac{r^{2}}{W^{2}}\right) \sin \phi,
\end{aligned}
$$

for the coupling between the transverse static magnetic field $\hat{B}_{y}^{(0)}$ and the GB, and we have

$$
\begin{aligned}
n_{x-x}^{(1)}= & \frac{1}{\mu_{0} \hbar \omega_{e}} \operatorname{Re}\left\langle\tilde{E}_{y}^{(1) *} \tilde{B}_{z}^{(0)}\right\rangle_{\omega_{e}=\omega_{g}}=\frac{1}{\mu_{0} \hbar \omega_{e}} \\
& \cdot\left\{\frac{A_{x} \hat{B}_{z}^{(0)} \psi_{0} k_{g} \Delta z r}{2\left[1+(z / f)^{2}\right]^{\frac{1}{2}}\left(z+f^{2} / z\right)} \sin \mathscr{P}\right. \\
& \left.+\frac{A_{x} \hat{B}_{z}^{(0)} \psi_{0} \Delta z r}{W_{0}^{2}\left[1+(z / f)^{2}\right]^{\frac{3}{2}}} \cos \mathscr{P}\right\}
\end{aligned}
$$

$$
\cdot \exp \left(-\frac{r^{2}}{W^{2}}\right) \sin \phi
$$

for the coupling between the longitudinal static magnetic field $\hat{B}_{z}^{(0)}$ and the GB.

Equations (B.11) and (B.12) show that the signal photon fluxes received in this direction would be the PPFs generated by the pure $\otimes$-type polarization (the tensor-mode gravitons) and by the pure $x$-type polarization (the vector-mode gravitons), respectively.

Since $n_{x}^{(0)}=0$, the noise photon flux fluctuation in the displaying condition, Eq. (B.8), will not be caused by the BPF of this direction, but would be caused by other noise photon fluxes. However, the latter are much less than the former [44] in the 3DSR. In this case, the displaying condition in Table 2 can be greatly relaxed. In other words, the PPFs produced by the HFGWs in the braneworld [21] and in the short-term anisotropic inflation [32], may almost be instantaneously displayed, and the requisite minimal accumulation time of the signal displaying the HFGWs predicted by the interaction [33] of astrophysical plasma with intense EM waves, by the pre-big-bang [27,28] and by the quintessential inflationary [29-31] models, would be further relaxed effectively. Thus, utilizing a highly orientational receiving surface to display the PPFs will be very useful.

By the way, the wave impedance and wave impedance matching would also play important roles for the displaying condition. For most of the signal photon fluxes discussed in this paper, the ratios of the electric components to the magnetic components are much less than that of the background noise photon fluxes. The typical values of the wave impedance of the former are $\sim 10^{-4} \Omega$ or less, and the typical values of the latter are $\sim 100 \Omega$ to $377 \Omega[43,59]$. This means that the 3DSR system would be equivalent to a "good superconductor" as regards the PPFs. Thus, the PPFs could be distinguished from the BPF and other noise photons by the wave impedance matching, and this is another important symbol to distinguish them.

It is very interesting to compare the PPFs $n_{x-\otimes}^{(1)}, n_{x-x}^{(1)}$ propagating along the $x$-direction and the $\operatorname{PPF} n_{y-\oplus, b, l}^{(1)}$ propagating along the $y$-direction. Notice that in the $x$-direction $n_{x}^{(0)}=0$ (the dominant noise photon flux in the $x$-direction caused by the GB) [see Eq. (B.10)], while $n_{y}^{(0)}$ (the dominant noise photon flux in the $y$-direction caused by the GB) has a non-vanishing value (see below), thus the displaying condition of the PPFs $n_{x-\otimes}^{(1)}, n_{x-x}^{(1)}$, Eqs. (B.11), Eqs. (B.12), is much better than that of $n_{y-\oplus, b, l}^{(1)}$, but distinguishing and displaying $n_{y-\oplus, b, l}^{(1)}$ are still possible due to the very different physical behavior between $n_{y-\oplus, b, l}^{(1)}$ and $n_{y}^{(0)}$ (see below). 
From Eq. (64) and Eqs. (A.2), (A.3), we have

$$
\begin{aligned}
n_{y-\oplus, b, l}^{(1)}= & \frac{1}{2 \mu_{0} \hbar \omega_{e}} \operatorname{Re}\left\langle\tilde{E}_{z}^{(0) *} \tilde{B}_{x}^{(1)}\right\rangle_{\omega_{e}=\omega_{g}}=\hat{B}_{x}^{(0)} k_{g} \Delta z x \\
& \cdot \frac{\left(A_{b}-A_{\oplus}+2 \sqrt{2} A_{l}\right)}{2 \mu_{0} \hbar \omega_{e}} \operatorname{Re}\left\langle F_{1}^{*}\right. \\
& \left.\cdot \exp \left[i\left(k_{g} z-\omega_{g} t\right)\right]\right\rangle_{\omega_{e}=\omega_{g}},
\end{aligned}
$$

and, $\quad n_{y}^{(0)}=\frac{1}{2 \mu_{0} \hbar \omega_{e}} \operatorname{Re}\left\langle\tilde{E}_{x}^{(0) *} \tilde{B}_{z}^{(0)}\right\rangle_{\omega_{e}=\omega_{g}}$

$$
=\frac{\psi_{0}^{2} y}{2 \mu_{0} \hbar \omega_{e}} \exp \left(-\frac{2 r^{2}}{W^{2}}\right)\left[\frac{k_{e}}{\omega_{e} \sqrt{1+(z / f)^{2}}}\right] \text {; }
$$

Eqs. (B.13) and (B.14) show the following important properties:

(i). $\left.n_{y-\oplus, b, l}^{(1)}\right|_{y=0}=n_{y-\oplus, b, l(\max )}^{(1)}$, while $\left.n_{y}^{(0)}\right|_{y=0}=0$,

i.e., the position (the longitudinal symmetrical surface of the GB) of peak value of the signal photon flux $n_{y-\oplus, b, l}^{(1)}$ is just the zero value area of the $\operatorname{BPF} n_{y}^{(0)}$ (the background noise photon flux). We have

(ii). $\quad n_{y-\oplus, b, l}^{(1)} \propto \exp \left(-\frac{r^{2}}{W^{2}}\right)=\exp \left(-\frac{x^{2}+y^{2}}{W^{2}}\right)$,

$$
n_{y}^{(0)} \propto \exp \left(-\frac{2 r^{2}}{W^{2}}\right)=\exp \left(-\frac{2\left(x^{2}+y^{2}\right)}{W^{2}}\right),
$$

i.e., the decay rate of $n_{y-\oplus, b, l}^{(1)}$ is obviously slower than that of $n_{y}^{(0)}$, although the peak value of $n_{y}^{(0)}$ is much larger than that of $n_{y-\oplus, b, l}^{(1)}$.

(iii). $n_{y-\oplus, b, l}^{(1)}[\mathrm{Eq}$. (B.13)] is an even function of the coordinates $y$; thus, $n_{y-\oplus, b, l}^{(1)}$ has the same propagating direction in the regions of $y>0$ and $y<0$. At the same time, $n_{y}^{(0)}$ (Eq. (B.14)) is an odd function of the coordinates $y$, so the propagating directions of $n_{y}^{(0)}$ are opposite in the regions of $y>0$ and $y<0$ [see Fig. 8]. These properties provide the distinguishability between $n_{y-\oplus, b, l}^{(1)}$ and $n_{y}^{(0)}$ [see Fig. 8].

Figure 8 shows that, although the peak of $n_{y}^{(0)}$ is much larger than that of $n_{y-\oplus, b, l}^{(1)}$ (the signal photon flux generated by the HFGWs in the braneworld [21]), it is always possible to find suitable positions, where $n_{y-\oplus, b, l}^{(1)}$ and $n_{y}^{(0)}$ are comparable and distinguishable, and $n_{y-\oplus, b, l}^{(1)}$ would be measurable. In this case, due to the background photon flux decays being faster than the signal photon flux, in the area around $y=0.25 m$, the $n_{y}^{(0)}$ already decays into a comparable level to $n_{y-\oplus, b, l}^{(1)}$, and in the further area, the background noise will decay into a lower level than the measurable signal photon flux. This is satisfactory.
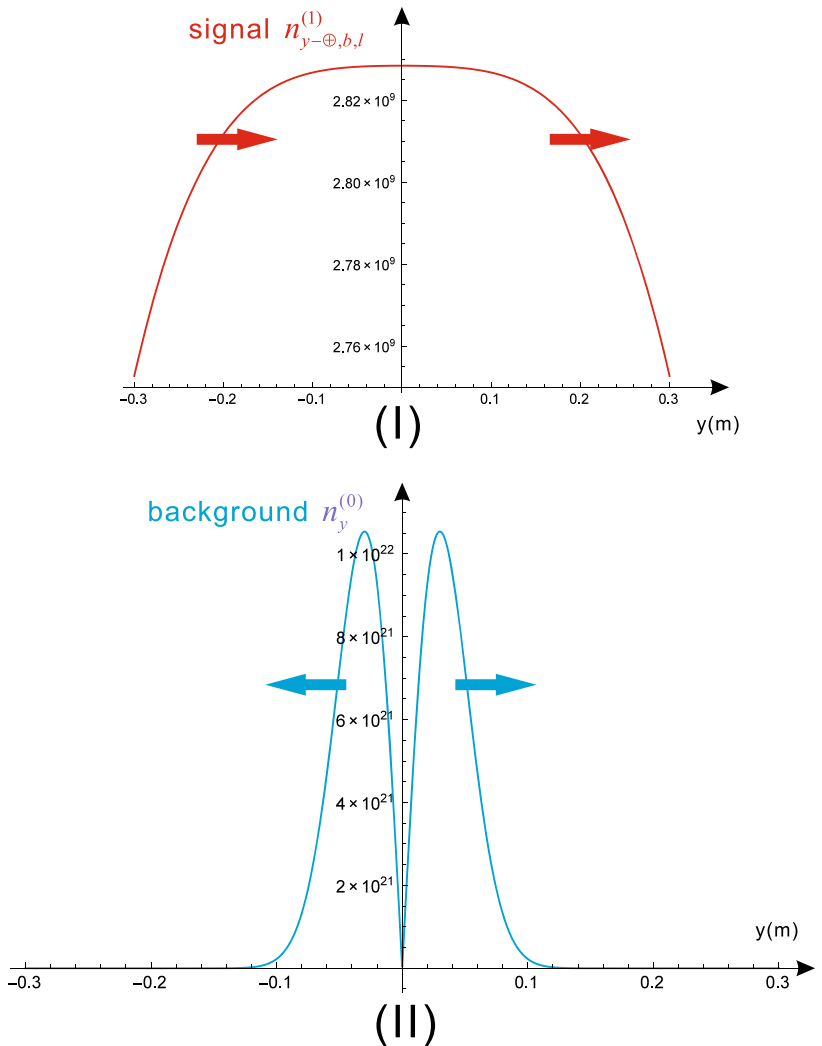

Fig. 8 Comparison of diagrams of the signal photon flux $n_{y-\oplus, b, l}^{(1)}$ (I) and background photon flux $n_{y}^{(0)}$ (II). It is shown that $n_{y-\oplus, b, l}^{(1)}$ propagates along the positive direction of the $y$-axis in the suitable resonance region, and the background noise photon flux $n_{y}^{(0)}$ propagates always in the opposite directions of the $y$-axis of $y>0$ and $y<0$

\section{Appendix C: The matching function between the HFGWs and the 3DSR system.}

Here we choose $n_{x-\otimes}^{(1)}$, Eq. (B.11), as an example to calculate the total signal photon flux propagating along the $x$-direction at effective receiving surface $\Delta s$. Then $n_{x-\otimes(\text { total })}^{(1)}$ can also be expressed as

$\left|n_{x-\otimes(\text { total })}^{(1)}\right|=\frac{A_{\otimes} \hat{B}_{y}^{(0)} \psi_{0}}{\mu_{0} \hbar \omega_{e}}\left|f\left(k_{g}, \mathbf{x}\right)\right|$,

where

$$
\begin{aligned}
f\left(k_{g}, \mathbf{x}\right)= & \int_{\Delta s}\left\{\frac{k_{g} \Delta z r}{2\left[1+(z / f)^{2}\right]^{\frac{1}{2}}\left(z+f^{2} / z\right)} \sin \mathscr{P}\right. \\
& \left.+\frac{\Delta z r \cos \mathscr{P}}{W_{0}^{2}\left[1+(z / f)^{2}\right]^{\frac{3}{2}}}\right\} \exp \left(-\frac{r^{2}}{W^{2}}\right) \sin \phi \mathrm{d} y \mathrm{~d} z
\end{aligned}
$$

is the matching function in Cartesian coordinate system, where $r=\sqrt{x^{2}+y^{2}}, \sin \phi=y / r=y / \sqrt{x^{2}+y^{2}}$. From 


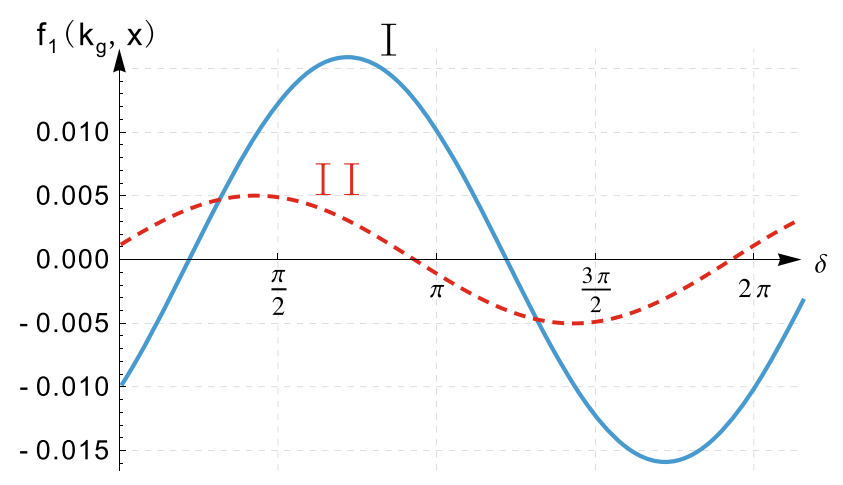

Fig. 9 Relation curves between the matching function $f\left(k_{g}, x\right)$ and the phase $\delta$. In fact, $\delta$ is the phase difference between the coherent nonstochastic HFGW and the GB in the 3DSR system. It is shown that, for the parameters $v_{e}=v_{g}=3 \times 10^{9} \mathrm{~Hz}, W_{0}=0.06 \mathrm{~m}, y \in[0 \mathrm{~m}, 0.1 \mathrm{~m}]$, $z \in[0,0.3 \mathrm{~m}]$, (i.e., $\Delta s=3.0 \times 10^{-2} \mathrm{~m}^{2}$ ) and the matching function $f\left(k_{g}, x\right)$ at the longitudinal symmetrical surface (i.e., the surface of $x=0), \delta=18 \pi / 25$ (or 2.26195) is the optimal phase, and $f\left(k_{g}, \mathbf{x}\right)=$ 0.0159 is the best matching function. For the matching function $f\left(k_{g}, x\right)$ at the surface of $x=10 \mathrm{~cm}$ ), $\delta=43 \pi / 100$ (or 1.35088) is the optimal phase, then $f_{1}\left(k_{g}, x\right)=0.00502$ is the best matching function

Eq. (C.18), we have

$\left|n_{x-\otimes(\text { total })}^{(1)}\right|_{\max }=\frac{A_{\otimes} \hat{B}_{y}^{(0)} \psi_{0}}{\mu_{0} \hbar \omega_{e}}\left|f\left(k_{g}, \mathbf{x}\right)\right|_{\max }$,

where $\left|n_{x-\otimes(\text { total })}^{(1)}\right| \max$ corresponds to the best coupling state. For the typical parameters of the 3DSR system, $A_{\otimes}=10^{-23}$, $v_{g}=3 \times 10^{9} \mathrm{~Hz}$ (the amplitude and frequency of HFGWs in the braneworld [21]); $\hat{B}_{y}^{(0)}=10 T, \psi_{0}=1.95 \times 10^{3} \mathrm{Vm}^{-1}$ (for the GB with $p=10 W, W_{0}=0.06 \mathrm{~m}, y \in[0,0.1 \mathrm{~m}], z \in$ $[0,0.3 \mathrm{~m}]$, the relation curve between the matching function $f_{1}\left(k_{g}, x\right)$ and the phase $\delta$ can be shown in Fig. 9 (at the surfaces of $x=0$ and at the surface of $x=10 \mathrm{~cm}$ ), i.e., the curves I and II, respectively.

For such coherent non-stochastic constant amplitude HFGW, the optimal phase is $\delta=18 \pi / 25$ (or 2.26195), then $\left|f\left(k_{g}, \mathbf{x}\right)\right|=\left|f\left(k_{g}, \mathbf{x}\right)\right|_{\max }=0.0159$, at the longitudinal symmetrical surface of $x=0$, and $\left|n_{x-\otimes(\text { total })}^{(1)}\right|_{\max } \approx$ $1.21 \times 10^{9} s^{-1}$ at receiving surface $\Delta s \sim 3 \times 10^{-2} \mathrm{~m}^{2}$.

For the stochastic HFGWs (e.g., the relic HFGWs in the pre-big-bang $[27,28]$ or in the quintessential inflationary [29-31]), it is necessary to integrate over all possible phases. Notice that this integration is not equal to zero and it is only limited in the positive value region or in the negative value region (which correspond to two opposite propagating directions of $n_{x-\otimes}^{(1)}$ ) due to the high directivity of the signal photon flux $n_{x-\otimes}^{(1)}$ and suitable orientation of the receiving surface. For the relic HFGW $\left(v_{g}=3 \times 10^{9} \mathrm{~Hz}, h \sim 10^{-29}\right)$ in the pre-big-bang, $\left|n_{x-\otimes(\text { total })}^{(1)}\right| \max \approx 10^{3} s^{-1}$, and for the relic HFGW $\left(v_{g}=3 \times 10^{9} \mathrm{~Hz}, h \sim 10^{-30}\right)$ in the quintessential inflationary, $\left|n_{x-\otimes(\text { total })}^{(1)}\right|_{\max } \approx 10^{2} s^{-1}$ (see Table 2 ).
In this case, the zero value characteristics of the background noise photo flux BPFs $n_{x}^{(0)}, n_{y}^{(0)}$ at the longitudinal symmetric surface, and the peak property of the signal photon fluxes $\left(n_{x-\otimes}^{(1)}, n_{x-\otimes}^{(2)}\right)$ at the longitudinal symmetric surface, inspire us to utilize the fractal membranes (they are very effective microwave lenses with a strong focusing function and the "one-way valve" property $[41,42,62-64]$ to the photon fluxes in the $\mathrm{GHz}$ to $\mathrm{THz}$ band), and they will provide a better way of selecting for the signal photon fluxes, especially for the signal photon fluxes generated by the stochastic relic HFGWs. These issues will also be discussed and studied in detail elsewhere.

\section{References}

1. B.P. Abbott et al. (LIGO Scientific Collaboration and Virgo Collaboration), Phys. Rev. Lett. 116, 061102 (2016a)

2. B.P. Abbott et al. (LIGO Scientific Collaboration and Virgo Collaboration), Phys. Rev. Lett. 116, 241103 (2016b)

3. B.P. Abbott et al. (LIGO Scientific Collaboration and Virgo Collaboration), Phys. Rev. Lett. 118, 221101 (2017a) https://doi.org/ 10.1103/PhysRevLett.118.221101

4. B.P. Abbott et al., Astrophys. J. Lett. 851, L35 (2017b)

5. B.P. Abbott et al. (LIGO Scientific Collaboration and Virgo Collaboration), Phys. Rev. Lett. 119, 141101 (2017c)

6. B.P. Abbott et al. (LIGO Scientific Collaboration and Virgo Collaboration), Phys. Rev. Lett. 119, 161101 (2017d)

7. B.P. Abbott et al. (LIGO Scientific Collaboration and Virgo Collaboration), arXiv:1811.12907 [astro-ph.HE]

8. B.P. Abbott et al. (LIGO Scientific Collaboration and Virgo Collaboration), Phys. Rev. X 6, 041015 (2016c). https://doi.org/10.1103/ PhysRevX.6.041015

9. D. Andriot, G.L. Gomez, J. Cosmol. Astropart. Phys. 2017, 048 (2017). http://stacks.iop.org/1475-7516/2017/i=06/a=048

10. L. Visinelli, N. Bolis, S. Vagnozzi, Phys. Rev. D 97, 064039 (2018)

11. D.M. Eardley, D.L. Lee, A.P. Lightman, R.V. Wagoner, C.M. Will, Phys. Rev. Lett. 30, 884 (1973)

12. C.M. Will, Theory and experiments in gravitational physics (Cambridge University Press, Cambridge, 1993)

13. C. Brans, R.H. Dicke, Phys. Rev. 124, 925 (1961)

14. Y. Fujii, K. Maeda, The Scalar-tensor Theory of Gravitation (Cambridge University Press, Cambridge, 2002)

15. C. de Rham, L. Heisenberg, Phys. Rev. D 84, 043503 (2011). https://doi.org/10.1103/PhysRevD.84.043503

16. L. Heisenberg, R. Kimura, K. Yamamoto, Phys. Rev. D 89, 103008 (2014). https://doi.org/10.1103/PhysRevD.89.103008

17. J.B. Jimenez, L. Heisenberg, Phys. Lett. B 757, 405 (2016). https:// doi.org/10.1016/j.physletb.2016.04.017

18. S. Capozziello, M. Francaviglia, Gen. Relativ. Gravit. 40, 357 (2008). https://doi.org/10.1007/s10714-007-0551-y

19. G. Dvali, G. Gabadadze, M. Porrati, Phys. Lett. B 485, 208 (2000)

20. S.S. Seahra, C. Clarkson, R. Maartens, Phys. Rev. Lett. 94, 121302 (2005)

21. C. Clarkson, S.S. Seahra, Class. Quantum Gravity 24, F33 (2007). https://doi.org/10.1088/0264-9381/24/9/F01

22. A. Nishizawa, A. Taruya, K. Hayama, S. Kawamura, M.-A. Sakagami, Phys. Rev. D 79, 082002 (2009). https://doi.org/10.1103/ PhysRevD.79.082002

23. A. Nishizawa, K. Hayama, Phys. Rev. D 88, 064005 (2013). https:// doi.org/10.1103/PhysRevD.88.064005 
24. A. Nishizawa, A. Taruya, S. Kawamura, Phys. Rev. D 81, 104043 (2010)

25. L.P. Grishchuk, arXiv:gr-qc/0504018 (2005)

26. M.L. Tong, Y. Zhang, Phys. Rev. D 80, 084022 (2009). https://doi. org/10.1103/PhysRevD.80.084022

27. M. Gasperini, G. Veneziano, Phys. Rep. 373, 1 (2003). https://doi. org/10.1016/S0370-1573(02)00389-7

28. G. Veneziano, Sci. Am. 290, 54 (2004)

29. M. Giovannini, Phys. Rev. D 60, 123511 (1999). https://doi.org/ 10.1103/PhysRevD.60.123511

30. M. Giovannini, Class. Quantum Gravity 26, 045004 (2009). https:// doi.org/10.1088/0264-9381/26/4/045004

31. M. Giovannini, Class. Quantum Gravity 31, 225002 (2014)

32. A. Ito, J. Soda, J. Cosmol. Astropart. Phys. 2016, 035 (2016)

33. M. Servin, G. Brodin, Phys. Rev. D 68, 044017 (2003). https://doi. org/10.1103/PhysRevD.68.044017

34. W.-B. Han, S.-S. Xue, Phys. Rev. D 89, 024008 (2014)

35. H. Wen, F.-Y. Li, J. Li, Z.-Y. Fang, A. Beckwith, Chin. Phys. C 41, 125101 (2017)

36. J. Liu, Z.-K. Guo, R.-G. Cai, G. Shiu, Phys. Rev. Lett. 120, 031301 (2018). https://doi.org/10.1103/PhysRevLett.120.031301

37. R. Easther, E.A. Lim, J. Cosmol. Astropart. Phys. 2006, 010 (2006). http://stacks.iop.org/1475-7516/2006/i=04/a=010

38. X. Li, L.-L. Wang, J. Li, Eur. Phys. J. C 77, 638 (2017)

39. T. Fujita, K. Harigaya, M. Kawasaki, R. Matsuda, Phys. Rev. D 89, $103501(2014)$

40. F.Y. Li, M.X. Tang, D.P. Shi, Phys. Rev. D 67, 104008 (2003). https://doi.org/10.1103/PhysRevD.67.104008

41. F.Y. Li, R.M.L. Baker Jr., Z.Y. Fang, G.V. Stepheson, Z.Y. Chen, Eur. Phys. J. C 56, 407 (2008)

42. F.Y. Li et al., Phys. Rev. D 86, 064013 (2009). arXiv:0909.4118v2

43. F.Y. Li, H. Wen, Z.Y. Fang, L.F. Wei, Y.W. Wang, M. Zhang, Nuclear Physics B 911, 500 (2016). arXiv:1505.06546 [gr-qc]

44. R. Woods et al., J. Mod. Phys. 2, 498 (2011)

45. H. Wen, F.Y. Li, Z.Y. Fang, Phys. Rev. D 89, 104025 (2014)

46. R. Ballantini et al., Class. Quantum Gravity 20, 3505 (2003)

47. M. Goryachev, M.E. Tobar, Phys. Rev. D 90, 102005 (2014). https:// doi.org/10.1103/PhysRevD.90.102005
48. L.P. Grishchuk, arXiv:gr-qc/0306013 (2003)

49. F.Y. Li, R.M.L. Baker Jr., Int. J. Mod. Phys. B 21, 3274 (2007), 6th International Conference on New Theories, Discoveries and Applications of Superconductors and Related Materials, Sydney, Australia, Jan. 09-11, 2007. https://doi.org/10.1142/ S0217979207044366

50. D. Boccaletti, V. De Sabbata, P. Fortint, C. Gualdi, Nuovo Cim. B 70, 129 (1970). https://doi.org/10.1007/BF02710177

51. W.K. DeLogi, A.R. Mickelson, Phys. Rev. D 16, 2915 (1977). https://doi.org/10.1103/PhysRevD.16.2915

52. L.M. Widrow, Rev. Mod. Phys. 74, 775 (2002)

53. J.C. Brown, M. Haverkorn, B.M. Gaensler, A.R. Taylor, N.S. Bizunok, N.M. McClure-Griffiths, J.M. Dickey, A.J. Green, Astrophys. J. 663, 258 (2007). http://stacks.iop.org/0004-637X/663/ $\mathrm{i}=1 / \mathrm{a}=258$

54. R.D. Nan, D. Li, C.J. Jin et al., Int. J. Mod. Phys. D 20, 989 (2011). https://doi.org/10.1142/S0218271811019335

55. D. Li, Z. Pan, Radio Sci. 51, 1060 (2016). https://doi.org/10.1002/ 2015RS005877

56. J.L. Han, R.N. Manchester, A.G. Lyne, G.J. Qiao, W. van Straten, Astrophys. J. 642, 868 (2006)

57. A. Yariv, Quantum electronics (Wiley, 1989).http://books.google. co.uk/books?id=UTWg1VIkNuMC

58. G.V. Stephenson, AIP Conf. Proc. 1103, 542 (2009)

59. J.C. Haslett, Essentials of radio wave propagation, The Cambridge wireless essentials series (McGraw-Hill, Cambridge, 2008)

60. Y.F. Tan, F.T. Wang, Z.M. Chen, Y.N. Pan, G.L. Kuang, Supercond. Sci. Technol. 22, 025010 (2009)

61. D. Li, R. Nan, Z. Pan, Proc. Int. Astron. Union 8, 325 (2012)

62. S. Anwar, S. Li, S. Li, Q. Duan, W. Lu, B. Hou, Appl. Phys. A 121, $1061(2015)$

63. W.J. Wen, L. Zhou, J. Li, W.K. Ge, C.T. Chan, P. Sheng, Phys. Rev. Lett. 89, 223901 (2002). https://doi.org/10.1103/PhysRevLett.89. 223901

64. L. Zhou, W.J. Wen, C.T. Chan, P. Sheng, Appl. Phys. Lett. 82, 1012 (2003). https://doi.org/10.1063/1.1553993 